\title{
Putative Cell Adhesion Membrane Protein Vstm5 Regulates Neuronal Morphology and Migration in the Central Nervous System
}

\author{
A-Ram Lee, ${ }^{1,2} \odot$ Kwang Woo Ko, ${ }^{1,4}$ Hojae Lee, ${ }^{1}$ Yi-Seul Yoon, ${ }^{1,2,3}$ Mi-Ryoung Song, ${ }^{1}$ and ${ }^{\circledR C h u l-S e u n g ~ P a r k ~}{ }^{1,2,3}$ \\ ${ }^{1}$ School of Life Sciences, ${ }^{2}$ National Leading Research Laboratory, and ${ }^{3}$ GIST Research Institute (GRI), Gwangju Institute of Science and Technology (GIST), \\ Gwangju, 500-712, Republic of Korea, and ${ }^{4}$ Department of Developmental Biology, Washington University School of Medicine, St. Louis, Missouri 63110
}

During brain development, dynamic changes in neuronal membranes perform critical roles in neuronal morphogenesis and migration to create functional neural circuits. Among the proteins that induce membrane dynamics, cell adhesion molecules are important in neuronal membrane plasticity. Here, we report that V-set and transmembrane domain-containing protein 5 (Vstm5), a cell-adhesion-like molecule belonging to the Ig superfamily, was found in mouse brain. Knock-down of Vstm5 in cultured hippocampal neurons markedly reduced the complexity of dendritic structures, as well as the number of dendritic filopodia. Vstm5 also regulates neuronal morphology by promoting dendritic protrusions that later develop into dendritic spines. Using electroporation in utero, we found that Vstm5 overexpression delayed neuronal migration and induced multiple branches in leading processes during corticogenesis. These results indicate that Vstm5 is a new cell-adhesion-like molecule and is critically involved in synaptogenesis and corticogenesis by promoting neuronal membrane dynamics.

Key words: dendrite; dendritic filopodia; dendritic spine; morphogenesis; neuronal migration; Vstm5

\section{Significance Statement}

Neuronal migration and morphogenesis play critical roles in brain development and function. In this study, we demonstrate for the first time that V-set and transmembrane domain-containing protein 5 (Vstm5), a putative cell adhesion membrane protein, modulates both the position and complexity of central neurons by altering their membrane morphology and dynamics. Vstm 5 is also one of the target genes responsible for variations in patient responses to treatments for major depressive disorder. Our results provide the first evidence that Vstm5 is a novel factor involved in the modulation of the neuronal membrane and a critical element in normal neural circuit formation during mammalian brain development.

\section{Introduction}

During brain development, dynamic changes of the cellular membrane coordinated with the cytoskeleton are essential for axonal development, dendritic branching, synaptic plasticity, and neuronal migration (Luo, 2002). In early neuronal differentiation, actin-based structures such as lamellipodia and filopodia are important for the initial sprouting of neurites, which will later become axons and dendrites (da Silva and Dotti, 2002). Filopodia serve to recognize the extracellular environment and transfer signals into the cell by acting

Received Feb. 11, 2016; revised June 25, 2016; accepted Aug. 16, 2016.

Author contributions: A.-R.L., K.W.K., H.L., M.-R.S., and C.-S.P. designed research; A.-R.L., K.W.K., Y.-S.Y., and H.L. performed research; A.-R.L. and C.-S.P. analyzed data; A.-R.L., K.W.K., M.-R.S., and C.-S.P. wrote the paper.

This work was supported by National Leading Research Laboratories (Grant 2011-0028665), the Korea National Research Foundation, and the GIST Research Institute (GRI) in 2016.

The authors declare no competing financial interests.

Correspondence should be addressed to Chul-Seung Park, Ph.D., Professor, School of Life Sciences, Principal Investigator, National Leading Research Laboratory, Gwangju Institute of Science and Technology (GIST), 261 Cheomdan-gwagiro, Buk-gu, Gwangju, 500-712, Republic of Korea. E-mail: cspark@gist.ac.kr.

DOI:10.1523/JNEUROSCI.0541-16.2016

Copyright $\odot 2016$ the authors $\quad 0270-6474 / 16 / 3610181-17 \$ 15.00 / 0$ as "antennae" (Mattila and Lappalainen, 2008) and are also crucial for neurite initiation and neuritogenesis (Dent et al., 2007).

Filopodial structures filled with actin filament bundles rapidly alter position and shape during neuronal development (PorteraCailliau et al., 2003). In dendrites, filopodia are long and thin protrusions without bulbous heads and play critical roles in spinogenesis and synaptogenesis (Ziv and Smith, 1996; Bhatt et al., 2009). According to the results from in vivo two-photon imaging experiments, spontaneously extended filopodia from dendrites search for silent presynaptic terminals. This dynamic behavior of dendritic filopodia induces multiple synaptic contacts with neighboring axons during early postnatal development and the resulting spines undergo highly selective maturation (Ziv and Smith, 1996; Fiala et al., 2002; Zuo et al., 2005; Bhatt et al., 2009). During corticogenesis, the migration of newly generated neurons is closely associated with membrane protrusions such as filopodia. An increase in membrane protrusions inhibits migration, whereas a decrease enhances neuronal migration (Carlson and Soderling, 2009; Guerrier et al., 2009). 
Membrane proteins located on the cell surface transfer extrinsic signals from the environment and trigger diverse intracellular signaling pathways linked to the actin cytoskeleton (Chothia and Jones, 1997). In spinogenesis, most surface proteins, such as cadherins, ephrins, neurexins, nectins, SALMs, syndecan-2, and ICAM-5, induce dendritic filopodia and regulate spine development (Matsuno et al., 2006; Lin et al., 2007; Kayser et al., 2008; Xie et al., 2008; Yoshihara et al., 2009). In particular, cell adhesion molecules (CAMs) such as NCAM and N-cadherin interact with the surrounding environmental cues and play essential roles in neuronal migration in vivo (Kadowaki et al., 2007; Burgess et al., 2008). The molecular mechanisms responsible for the regulation of spinogenesis and neuronal migration by membrane proteins are well understood, but the roles of filopodia formation associated with morphogenesis, synaptogenesis, and neuronal migration are less well known.

To identify novel membrane proteins involved in the structural dynamics of the neuronal membrane, we screened potential membrane proteins expressed in the CNS and assayed their ability to alter membrane morphology. Among unannotated candidate genes, we focused on a gene encoding a putative CAM in mouse basal ganglia. The protein, called $\mathrm{V}$-set and transmembrane domain-containing 5 (Vstm5), is a member of the VSTM family.

We found that Vstm5 is an N-glycosylated membrane protein highly expressed in the CNS. It facilitated the formation of neuronal dendrites and protrusions such as dendritic filopodia. In synaptogenesis, Vstm 5 regulates synapse formation by altering dendritic spine morphology and actin distribution. Vstm5 also regulates neuronal morphogenesis and migration during cortical development in mouse brain. These results indicate that Vstm 5 may be a novel membrane protein directly regulating membrane dynamics for neuronal morphogenesis and migration and potentially regulates neural circuits and brain functions.

\section{Materials and Methods}

DNA constructs and molecular cloning. The full-length complementary DNA (cDNA) of the Vstm5 protein was purchased from CloneRanger (Invitrogen). Vstm 5 cDNA was amplified using PCR and then subcloned in-frame into pEGFP-N3 (Clonetech) or pcDNA3.1(+) for mammalian cell expression. The $\mathrm{C}$ terminus of Vstm 5 was also tagged with a monoclonal antibody epitope (Vstm5::1D4) by replacing EGFP in pEGFP-N3 with a C-terminal rhodopsin 1D4 tag (Hodges et al., 1988). To construct the C-terminal deletion mutants, Vstm5-CTD, Vstm5-C(C $\triangle 14)$, and Vstm5-C(N $\triangle 17)$ were subcloned into pEGFP-N3 tagged with the GFP or 1D4 epitope. To observe the detailed morphology of spines in neurons, Vstm 5 constructs were subcloned into pCAGIG (Addgene).

Antibodies. For detection of the Vstm5 protein, polyclonal antibodies were raised in rabbit against the peptide KRRYKLKESTTEEIEMKEVEC, which corresponds to the C-terminal sequence of mouse Vstm5. The antiVstm 5 antibody was generated by AbFrontier. The other antibodies used were as follows: rabbit polyclonal and mouse monoclonal anti-GFP (both from Abcam); mouse monoclonal anti-1D4 (Abcam); rabbit polyclonal anti-MAP2 (Santa Cruz Biotechnology); mouse monoclonal anti-neurofilament (American Research Products); mouse monoclonal anti-PSD-95 (Affinity BioReagents); rabbit polyclonal anti-synaptophysin (Zymed Laboratories, Invitrogen); and Alexa Fluor-conjugated secondary antibodies (Alexa Fluor 488, Alexa Fluor 594, and Alexa Fluor 633; Invitrogen). Texas red-phalloidin (Invitrogen) was used to assess filamentous actin (F-actin).

Cell culture and transfection. COS-7 and HEK293 cells were cultured at $37^{\circ} \mathrm{C}$ in DMEM (Hyclone) supplemented with $10 \%$ fetal bovine serum (Hyclone) in a humidified atmosphere containing 5\% $\mathrm{CO}_{2}$. Cells were transfected using Lipofectamine 2000 (Invitrogen) according to the manufacturer's instructions. Mouse hippocampal neuronal cultures were prepared as described previously (Chang and De Camilli, 2001).
Briefly, hippocampi were dissected from embryonic day 18 (E18) mice and dissociated with papain (Worthington Biochemical). The cells were plated on poly-D-lysine-coated cover glasses at a density of $5 \times 10^{5}$ cells per $60 \mathrm{~mm}$ plastic dish and maintained in Neurobasal medium (Invitrogen) supplemented with B-27 (Invitrogen) and 2 mM L-GlutaMAX (Invitrogen). Neurons were transfected at din vitro (DIV) 5 or DIV10 using a modified calcium-phosphate precipitation method (Ryan et al., 2005).

$R N A$ extraction and semiquantitative RT-PCR. Mice were humanely killed and their tissues (brain, lung, liver, stomach, heart, kidney, spleen, small intestine, large intestine, bladder, and spinal cord) were isolated. The CNS was further divided into subregions (olfactory bulb, thalamus, hippocampus, cerebrum, midbrain, cerebellum, and spinal cord). Total RNAs were purified from isolated tissues using TRIzol reagent (Molecular Research Center). Each tissue was homogenized using a glass Teflon homogenizer and subsequent extraction steps were performed as described in the manufacturer's instructions. cDNA was synthesized from total RNA by priming with oligo $(\mathrm{dT})_{12-18}$ (Invitrogen) using Moloney murine leukemia virus reverse transcriptase (Invitrogen) at $37^{\circ} \mathrm{C}$ for $1 \mathrm{~h}$. The transcripts of Vstm5 gene was amplified to generate specific fragments using a set of primers (forward 5' ${ }^{\prime}$ TCACAGGGAGCAGTTG AGTG-3', reverse $5^{\prime}$-TTGCTGATCGAATGGATGAA-3'). Mouse GAPDH primers (forward 5' ${ }^{\prime}$ TCCGTGTTCCTACCCCCAATG-3' ${ }^{\prime}$, reverse $5^{\prime}$-GGGAGTTGCTGTTGAAGTCGC- ${ }^{\prime}$ ) were used as an internal control for RT-PCR and real-time PCR (qRT-PCR) to normalize for differences in RNA preparations between different tissues.

Deglycosylation of Vstm5 using tunicamycin and enzymes. Cells were transiently transfected with Vstm 5 constructs and, $6 \mathrm{~h}$ after transfection, tunicamycin was added to the medium at various concentrations $(0,0.5$, 1.0 , and $5.0 \mu \mathrm{g} / \mathrm{ml}$ ). Cells were harvested $4 \mathrm{~h}$ after the addition of tunicamycin using RIPA buffer (20 mM HEPES, $150 \mathrm{~mm} \mathrm{NaCl}, 1$ mm EDTA, 1 mm EGTA, 1\% sodium deoxycholic acid, 1\% Triton X-100, 2 mм $\mathrm{Na}_{3} \mathrm{VO}_{4}, 1 \% \mathrm{NP}-40,2 \mathrm{~mm} \mathrm{NaF}$, and $1 \times$ protease inhibitor mixture; Complete, Roche Diagnostics). For the deglycosylation of Vstm5 using enzymes, the cells were treated with N-glycosidase/PNGase F (New England Biolabs) according to the manufacturer's instructions. The effects of chemical and enzymatic treatments on Vstm5 glycosylation were analyzed using SDS-PAGE, followed by immunoblotting. For chemical cross-linking, a monolayer and a single-cell suspension of Vstm5expressing cells were incubated in PBS containing $5 \mathrm{~mm}$ BS3 (Pierce) at room temperature for $15 \mathrm{~min}$. The reaction was quenched with the addition of $20 \mathrm{~mm}$ Tris- $\mathrm{HCl}$ at $\mathrm{pH} 7.5$ for $15 \mathrm{~min}$. After lysis for $30 \mathrm{~min}$, the cells were treated with $\mathrm{N}$-glycosidase/PNGase F and then subjected to Western blot analysis.

Coimmunoprecipitation and Western blot analysis. Transfected cells were gently lysed with lysis buffer $(25 \mathrm{~mm}$ Tris- $\mathrm{HCl}, \mathrm{pH} 7.5,150 \mathrm{~mm}$ $\mathrm{NaCl}, 1 \%$ sodium deoxycholic acid, $1 \%$ Triton X-100, 0.1\% SDS, $1 \mathrm{~mm}$ phenylmethylsulfonyl fluoride, and $1 \times$ protease inhibitor mixture; Complete, Roche Diagnostics) for $30 \mathrm{~min}$ on ice. Collected immunoprecipitates were incubated with a rabbit anti-GFP or mouse anti-1D4 antibody overnight at $4^{\circ} \mathrm{C}$, followed by the addition of Protein A Plus Agarose ( $20 \mu \mathrm{l}$; Thermo Scientific) for $1 \mathrm{~h}$ at $4^{\circ} \mathrm{C}$. The bound proteins were eluted and separated using SDS-PAGE (10-15\% gels) and transferred to PVDF membrane (Whatman). The membranes were blocked with $5 \%$ skim milk prepared in $1 \times$ TBST $(10 \mathrm{~mm}$ Tris- $\mathrm{HCl}, 100 \mathrm{~mm} \mathrm{NaCl}$, and $0.1 \%$ Tween 20, pH 7.5) for $1 \mathrm{~h}$, washed, and incubated with the primary antibody at $4^{\circ} \mathrm{C}$ overnight. After extensive washing in $1 \times$ TBST, the membrane was incubated with a horseradish peroxidase-conjugated secondary antibody (Jackson ImmunoResearch Laboratories). Proteins were visualized with ECL reagent (GE Healthcare Life Sciences).

$R N A$ interference. The siRNA for Vstm 5 was designed from nucleotides 695-715 of the mouse Vstm 5 cDNA sequence. Based on the method described previously (Paddison et al., 2004), the miR-30-incorporated scramble and Vstm5 template oligonucleotides were designed and synthesized separately with miR-30 primers and the additions of an XhoI site at the $5^{\prime}$ end and an EcoRI site at the $3^{\prime}$ end. The Vstm5 siRNA target sequence was 5'-GCCGTGGCTGTGGTGCTAATC-3'. The annealed template was cloned into the XhoI-EcoRI sites of the pCAG-miR30 vector (Invitrogen). Control small hairpin RNA (shRNA, shScramble) was designed by random changes in the shVstm5 nucleotide composition (5'-GGTCCGGGTGCCGTCTTG- 
TAA-3'). A shVstm5-resistant Vstm5 construct (Vstm5 ${ }^{\text {sh resist }}$ ) was generated by introducing seven silent mutations into the shVstm 5 targeting sequence. The construct with the modified shVstm5 target sequence $5^{\prime}$ GCTGTAGCCGTTGTACTTATT-3' was used in the rescue experiments. For the Cdc42 and Rac1 knock-down study, the mouse 5'-TAGTGAAGCCACAGATGTA- $3^{\prime}$ target sequence for Cdc42 and the $5^{\prime}$-TAGTGAAGCCACAGATGTA-3' target sequence for Racl were used. To determine the detailed morphology of the knock-down cells, the pCAG-mir30 vector was modified by inserting IRES-GFP from the pCAGIG vector (Addgene) using the NotI and HindIII restriction enzymes.

Cdc42/Rac1 activation assay. Cell were lysed in lysis buffer $(25 \mathrm{~mm}$ Tris- $\mathrm{HCl}, \mathrm{pH} 7.5,150 \mathrm{~mm} \mathrm{NaCl}, 1 \%$ sodium deoxycholic acid, $1 \%$ Triton $\mathrm{X}-100,0.1 \%$ SDS, $1 \mathrm{~mm}$ phenylmethylsulfonyl fluoride, and $1 \times$ protease inhibitor mixture; Complete, Roche Diagnostics) for $30 \mathrm{~min}$ on ice. GSTPAK1-PBD beads $(10 \mu \mathrm{l})$ were added to cleared lysates $(500 \mu \mathrm{l})$ and incubated at $4^{\circ} \mathrm{C}$ for $1 \mathrm{~h}$. Beads were then washed in lysis buffer and $2 \times$ SDS loading buffer was added. Proteins were denatured for $10 \mathrm{~min}$ at $100^{\circ} \mathrm{C}$, loaded onto SDS-PAGE gels, and immunoblotted using Cdc42or Rac1-specific antibodies.

Immunofluorescence and confocal microscopy. COS-7 cells and hippocampal neurons were washed 3 times with $1 \times \mathrm{PBS}$ and then with $4 \%$ $\mathrm{PFA} /$ sucrose in PBS for $15 \mathrm{~min}$ at room temperature. After washing, the cells were permeabilized with $0.25 \%$ Triton X-100 in PBS for 5 min at room temperature and blocked with 5\% BSA in PBS for 30 min. The cells were incubated with primary antibody for $1 \mathrm{~h}$ and subsequently with fluorescence-conjugated secondary antibody for $45 \mathrm{~min}$. Fluorescence images were acquired using a Fluoview FV 1000 confocal laser-scanning microscope equipped with $100 \times$ and $60 \times$ oil-immersion objectives and capable of an additional $4 \times$ zoom. For live-cell imaging, transfected cells were incubated in HEPES-buffered Tyrode's solution containing the following (in mM): $119 \mathrm{NaCl}, 5 \mathrm{KCl}, 25 \mathrm{HEPES}$ buffer, $2 \mathrm{CaCl}_{2}$, and $2 \mathrm{MgCl}_{2}$ with $0.6 \%$ glucose, $\mathrm{pH} 7.4$. Confocal live images were acquired every $10 \mathrm{~s}$ for $10 \mathrm{~min}$.

Scanning electron microscopy. Mock and Vstm5-transfected cells were fixed using $4 \% \mathrm{PFA}, \mathrm{pH} 7.4$, for $12 \mathrm{~h}$ at $4^{\circ} \mathrm{C}$, and fixed cells were rinsed in cold $1 \times$ PBS. After rinsing, cells were dehydrated in a graded series of ethanol prepared in PBS $(50 \%, 60 \%, 70 \%, 80 \%, 90 \%, 95 \%$, and $100 \%$ ethanol) for $10 \mathrm{~min}$ each on ice and then rapidly dried. Dried samples were coated with $20 \mathrm{~nm}$ platinum and visualized. The scanning electron microscope micrographs were produced by the Korea Photonics Technology Institute.

Image analysis and quantification. Analysis and quantification of data were performed using ImageJ. In COS-7 cells, filopodia were quantified by measuring the average number and length of filopodia in $20 \mu \mathrm{m}$ at three different areas on each cell. Spiny neurons were selected randomly. Dendritic filopodia were identified as long $(>2 \mu \mathrm{m})$ protrusions along the dendrite. To classify spine morphology, spines were defined based on their length and the ratio of head diameter ( $\mathrm{dh}$ ) to neck diameter $(\mathrm{dn})$ as follows: mushroom $(\mathrm{dh} / \mathrm{dn}>1.3)$, thin $(\mathrm{dh} / \mathrm{dn} \leq 1.3)$, and stubby $(\mathrm{dh} /$ $\mathrm{dn}<1$ ) (Harris et al., 1992). In vivo dendrite images were captured with a $60 \times$ objective at $6 \times$ zoom using $1 \mu \mathrm{m} z$-stack intervals. Multiple dendrite images were collected and the dendritic protrusions were analyzed using the StackReg plugin for ImageJ. The dendrites of neurons were traced and measured using the NeuronJ plugin and dendrite complexity was analyzed with the Sholl analysis plugin for ImageJ. Layer $2 / 3$ dendrites were imaged in vivo with an epifluorescence microscope (Zeiss) using a $20 \times$ (numerical aperture, NA 0.7) objective. Dendrites were then traced using the ImageJ plugin NeuronJ. Dendritic spines were acquired with confocal microscopy using a $63 \times($ NA 1.4) objective at $6 \times$ zoom with $0.5 \mu \mathrm{m} z$-stack intervals. Spine length and head width measurements were conducted using ImageJ. Pairwise comparisons were performed using an unpaired, two-tailed Student's $t$ test. Data are presented as means \pm SEM.

In utero electrophoresis and immunohistochemistry. In utero electroporation was performed as described previously (Tabata and Nakajima, 2001) with some modifications. Plasmid DNA was injected at a concentration of $1 \mu \mathrm{g} / \mu \mathrm{l}$ in the lateral ventricles of the embryo at E14.5, followed by electroporation using a CUY21 electroporator (NEPA Gene) with 6 pulses of $30 \mathrm{~V}$ for $50 \mathrm{~ms}$ each at $950 \mathrm{~ms}$ intervals. The developing brains fixed with $4 \%$ PFA were sectioned coronally with a cryostat (Leica) at 16 $\mu \mathrm{m}$ for postnatal day $1(\mathrm{P} 1)$ and $60 \mu \mathrm{m}$ for P10-P11 embryos. The slices were treated with $1 \times$ PBST $(1 \times$ PBS containing $0.2 \%$ Triton X-100), blocked in buffer containing $2 \%$ goat serum and $0.1 \%$ Triton X-100 for $1 \mathrm{~h}$ and subsequently incubated with primary antibodies in blocking buffer at $4^{\circ} \mathrm{C}$ overnight. After several washes with $1 \times$ PBST, slices were treated with fluorescence-conjugated secondary antibodies for $1 \mathrm{~h}$. The nuclei of brain slices were visualized using mounting medium containing DAPI (Vectashield; Vector Laboratories).

\section{Results}

\section{Vstm 5 is a novel membrane protein}

The Vstm5 protein is a member of the VSTM family, a group of membrane proteins with an extracellular V-set domain and a single membrane-spanning region. The nucleotide and deduced amino acid sequences of mouse Vstm5, previously annotated as LOC69137, are shown in Figure 1A. To find the cross-species homology of Vstm5, we searched the orthologues of Vstm5 in various vertebrates. The phylogenic tree of the VSTM5 amino acids showed high homology in various vertebrates from fish to humans (Fig. 1B). Mouse Vstm5, encoded in four different exons (Fig. $1 C$ ), shares $>91 \%$ amino acid sequence identity with rat Vstm 5 and shares $78 \%$ identity with the human Vstm 5 protein. A multiple sequence alignment of these proteins shows that Vstm5 is a highly conserved protein in evolution (Fig. 1D).

\section{Vstm 5 is a glycoprotein highly expressed in the CNS and forms multimeric complexes}

Vstm5, a type I integral membrane protein composed of 199 amino acids, consists of an $\mathrm{N}$-terminal signal peptide, an Ig-V-set domain, a transmembrane domain, and a short intracellular region (Fig. 2A). We initially investigated the temporal expression patterns of Vstm5 in various mouse tissues using RT-PCR. In adult mouse tissues, Vstm5 was highly expressed in the CNS, as well as in the stomach, kidney, and small intestine (Fig. 2B). As determined using real-time PCR analyses, Vstm 5 is expressed in various subregions of the CNS and is especially highly expressed in the thalamus, hippocampus, cerebrum, midbrain, and spinal cord (Fig. 2C). In developing mouse brain, low levels of Vstm5 mRNA were detected on E11, rapidly increased to peak at P1, and then gradually decreased (Fig. 2D).

When expressed in COS-7 cells, the C-terminal rhodopsin 1D4 epitope-tagged Vstm5 (Vstm5::1D4) showed multiple protein bands in the region of $30-45 \mathrm{kDa}$ (Fig. $2 E$ ), which is much higher than the expected molecular weight of $20.5 \mathrm{kDa}$ including the 1D4 tag but excluding the signal peptide, suggesting that Vstm5 is extensively glycosylated. Indeed, amino acid analysis of Vstm5 predicted four potential N-linked glycosylation sites in its extracellular domain (N43, N87, N101, N108; Fig. 1F). Treatment with tunicamycin, an antibiotic blocking the synthesis of $\mathrm{N}$-linked oligosaccharide chains on glycoproteins, shifted the multiple protein bands to a single band of $\sim 20 \mathrm{kDa}$ in a dosedependent manner (Fig. 2E, left blot). Similar results were obtained when the Vstm5::1D4-expressing cell lysate was digested with peptide- $N$-glycosidase F (PNGase F), an enzyme that removes N-linked glycosylation (Fig. 2E, right blot).

Many previous studies have confirmed that membrane proteins are crucial for cell function and signal transduction because they regulate the monomer-multimer transition on the cell membrane (Woolf and Linderman, 2003; Niessen et al., 2011). To determine whether Vstm 5 multimerizes in a cellular environment, we coexpressed Vstm 5 with two different C-terminal tags, Vstm5::GFP and Vstm5::1D4. When the COS-7 cell lysate was 
A

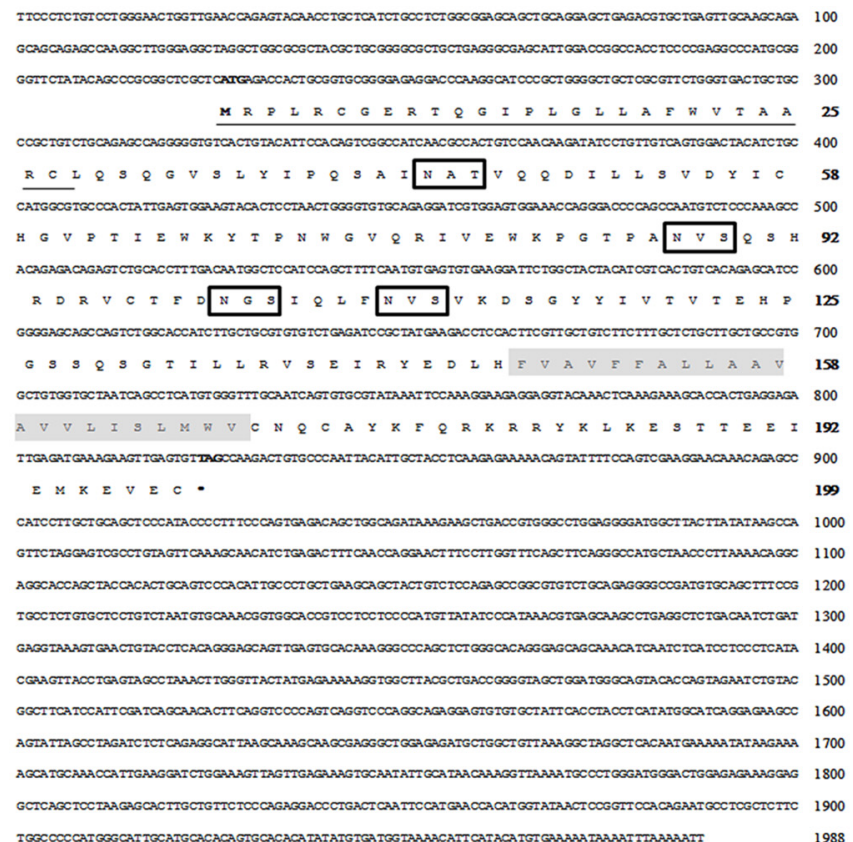

B

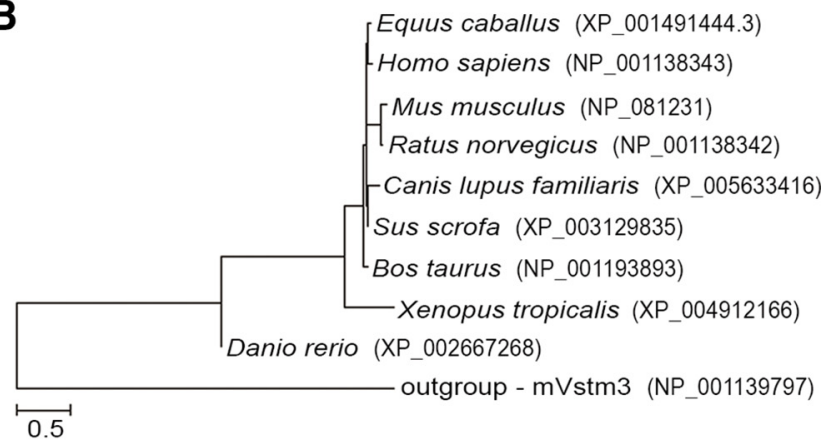

C

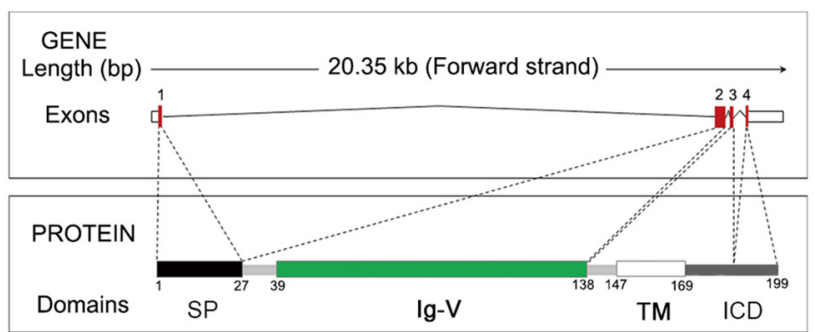

D

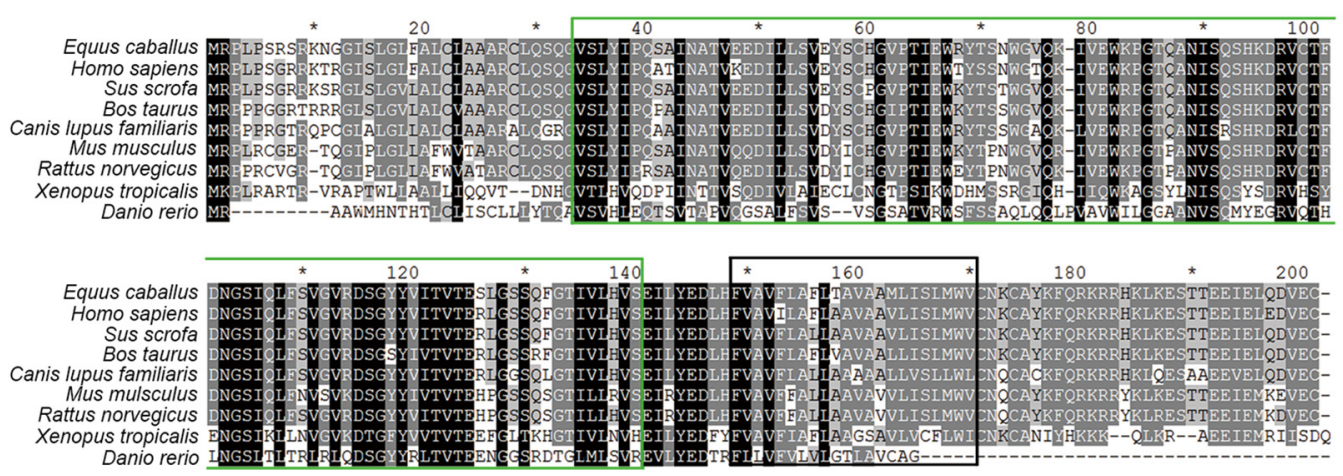

Figure 1. Vstm5 is a highly conserved membrane protein. A, Full-length predicted open reading frame of Vstm5 (GenBank NM_026955). The predicted amino acid sequence of Vstm5 is shown with the locations of $\mathrm{N}$-linked glycosylation sites and predicted transmembrane segments marked by rectangles and shading, respectively. Both nucleotides and amino acids are numbered at the right sides of their respective lines. The initiation ATG $(M)$ and stop codon TAG $\left(^{*}\right)$ are in bold font and the signal peptide regions are underlined. $\boldsymbol{B}$, Phylogenetic relationships of Vstm5 from Danio rerio to Homo sapiens were identified using MEGA6 software. The mouse Vstm3 was used for the outgroup (NP_001139797). C, Vstm5 gene arrangement and protein domain structure, which consists of a signal peptide (SP, black), Ig-like V-set domain (Ig-V, green), transmembrane domain (TM, white), and intracellular domain (ICD, dark gray). D, Whole sequences of Vstm5 from Equus caballus (XP_001491444.3), Homo sapiens (NP_001138343), Sus scrofa (XP_003129835), Bos taurus (NP_001193893), Canis lupus familiaris (XP_005633416), Mus musculus (NP_081231), Rattus norvegicus (NP_001138342), Xenopus tropicalis (XP_004912166), and Danio rerio (XP_002667268) were multiple aligned using Clustal X. The Ig-like vet domain and transmembrane domain are boxed in green and black, respectively.

pulled down using an anti-1D4 antibody, not only Vstm5::1D4 but also Vstm5::GFP was detected in the immunoprecipitate (Fig. $2 G$ ), suggesting potential homophilic multimerization of Vstm5. To determine the multimerization status of the Vstm5 protein on the cell surface, COS-7 cells expressing Vstm5::1D4 were separated on SDS-polyacrylamide gels under reducing and nonreducing conditions. Whereas the monomeric form of Vstm5 was observed in the presence of $\beta$-mercaptoethanol, at least four Vstm5 bands were discernible in the absence of the reducing agent (Fig. 2H, left blot). We also tried to cross-link Vstm5::1D4 in the cellular environment with BS3, a noncleavable membraneimpermeable cross-linker. In the absence of BS3, only a single protein band was observed after PNGase F treatment, corresponding to the molecular weight of deglycosylated monomeric Vstm5 at $\sim 22 \mathrm{kDa}$. With increasing concentrations of BS3, protein bands were identified near sizes corresponding to those with multiple integers of Vstm5, for example, 44, 66, and $88 \mathrm{kDa}$ (Fig. $2 \mathrm{H}$, right blot). We further examined whether Vstm 5 protein formed oligomers with cis- or trans-configurations on the cell surface. As shown in Figure 2I, COS-7 cells in an adherent monolayer and single cell suspension expressing either Vstm5::ID4 (Fig. 2I, left blot) or Vstm5::GFP (Fig. 2I, right blot) were treated with BS3, respectively, and then their multimeric status was examined using Western blot analysis. The band patterns and intensities were similar in both adherent and suspended cells, indicating that multimerization of Vstm5 homomers occurs mainly, if not exclusively, through cis interactions within the same cell membrane rather than across neighboring cells. Therefore, these results indicate that Vstm5 is an N-linked glycosylated protein mainly expressed in the CNS and it can form homomultimeric complexes in a cis configuration. 
B

A
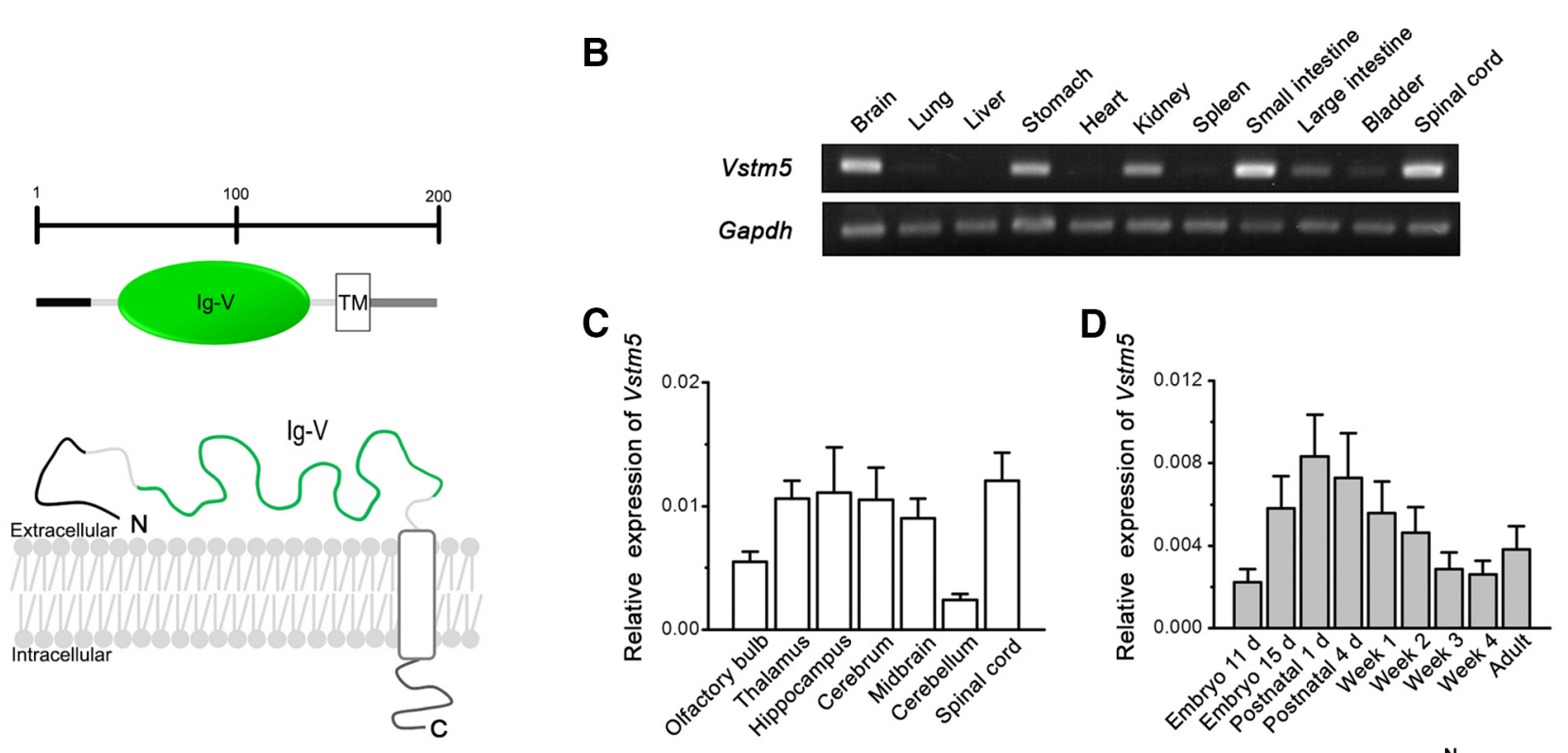

E

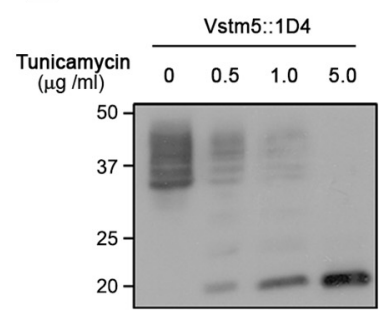

G

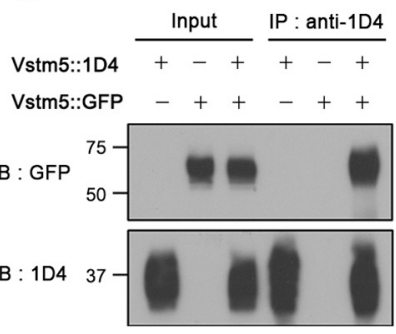

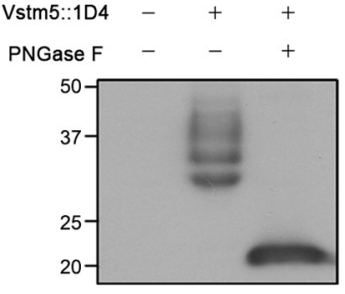

F

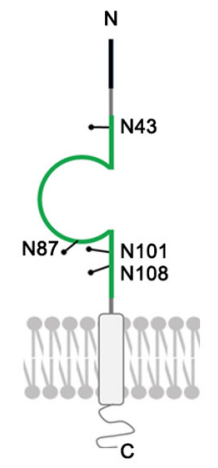

H

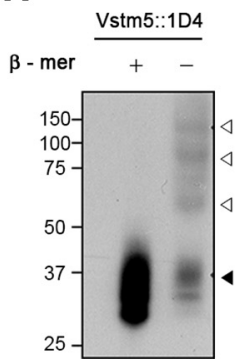

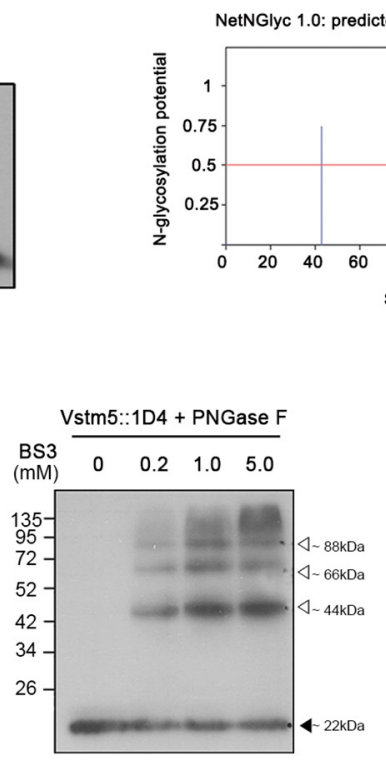

I

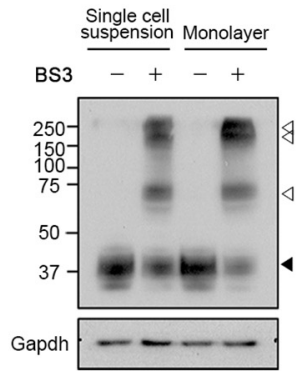

Figure 2. Vstm5 is an N-linked glyco-transmembrane protein expressed mainly in the CNS. A, Schematic illustration of the domain structure (top) and membrane topology (bottom) of Vstm5 analyzed using the protein database Simple Modular Architecture Tool (SMART). Black, Signal peptide; green, IgV-set domain (Ig-V); white, transmembrane domain; dark gray, intracellular domain. $B$, Reverse transcriptase $P C R$ results of Vstm $5 \mathrm{mRNA}$ expression in various mouse tissues. $C$, Vstm5 mRNA expression determined by real-time quantitative RT-PCR in adult mouse brain subregions and spinal cord. D, Vstm5 mRNA expression during neuronal development in mouse brain. Vstm5 mRNA expression normalized to the internal housekeeping gene mouse GAPDH. Mean and SEM were calculated from three independent samples per time point. E, Vstm5::1D4-transfected COS-7 cells were treated with tunicamycin at the indicated concentration for $6 \mathrm{~h}$ after transfection and the loaded proteins are shown in the blot on the left. Proteins resulting from a lysate digest without (-) or with (+) PNGase F (right blot). Western blotting was performed using anti-1D4 antibody. $\boldsymbol{F}$, Graph of predicted N-glycosylation sites in Vstm5 amino acid sequences using the NetNGlyc 1.0 server (Center for Biological Sequence Analysis; left image). Membrane topology and schematic illustration of N-glycosylated Vstm5 are shown. Black lines with filled circles indicate glycosylation sites (N43, N87, N101, and N108; right image). G, Vstm5::GFP and Vstm5::1D4 cotransfected or single-transfected lysates were immunoprecipitated with anti-1D4 antibody and subjected to Western blotting using anti-GFP or anti-1D4 antibodies. $\boldsymbol{H}$, Vstm5::1D4-transfected cell lysates were lysed in SDS sample buffer in the presence $(+)$ or absence $(-)$ of $\beta$-mercaptoethanol (left blot). Vstm5::1D4-expressing cells were treated with increasing concentrations of the cross-linker BS3 and then with PNGase F (right blot). I, Monolayer and single-cell suspensions expressing Vstm5::1D4 (left blot) or Vstm5::GFP (right blot) incubated in the absence ( - ) and presence (+) of $5 \mathrm{~mm}$ BS3. Protein samples were subjected to Western blotting using anti-1D4 or anti-GFP and anti-mGAPDH antibodies. Solid and open arrowheads indicate the monomer and oligomer forms of the Vstm5 protein, respectively.

Vstm5 induces filopodial protrusions in various cell types To characterize its cellular functions, Vstm5 was expressed exogenously in cell lines of different origins. When GFP-fused Vstm5 (Vstm5::GFP) was expressed, more numerous membrane protrusions were induced throughout the membrane edge of all cell lines tested, including those of neuronal (HT22, SH-SY5Y, and
Neuro-2a) and non-neuronal (HeLa) origins (Fig. 3E-H) than in GFP-expressing cells (Fig. $3 A-D$ ). The induction of protrusion formation was further examined using scanning electron microscopy. Whereas control COS-7 cells (Mock) showed only short projections (Fig. 3I), Vstm5::GFP-transfected cells showed numerous filopodia-like protrusions with long, thin shapes at the 

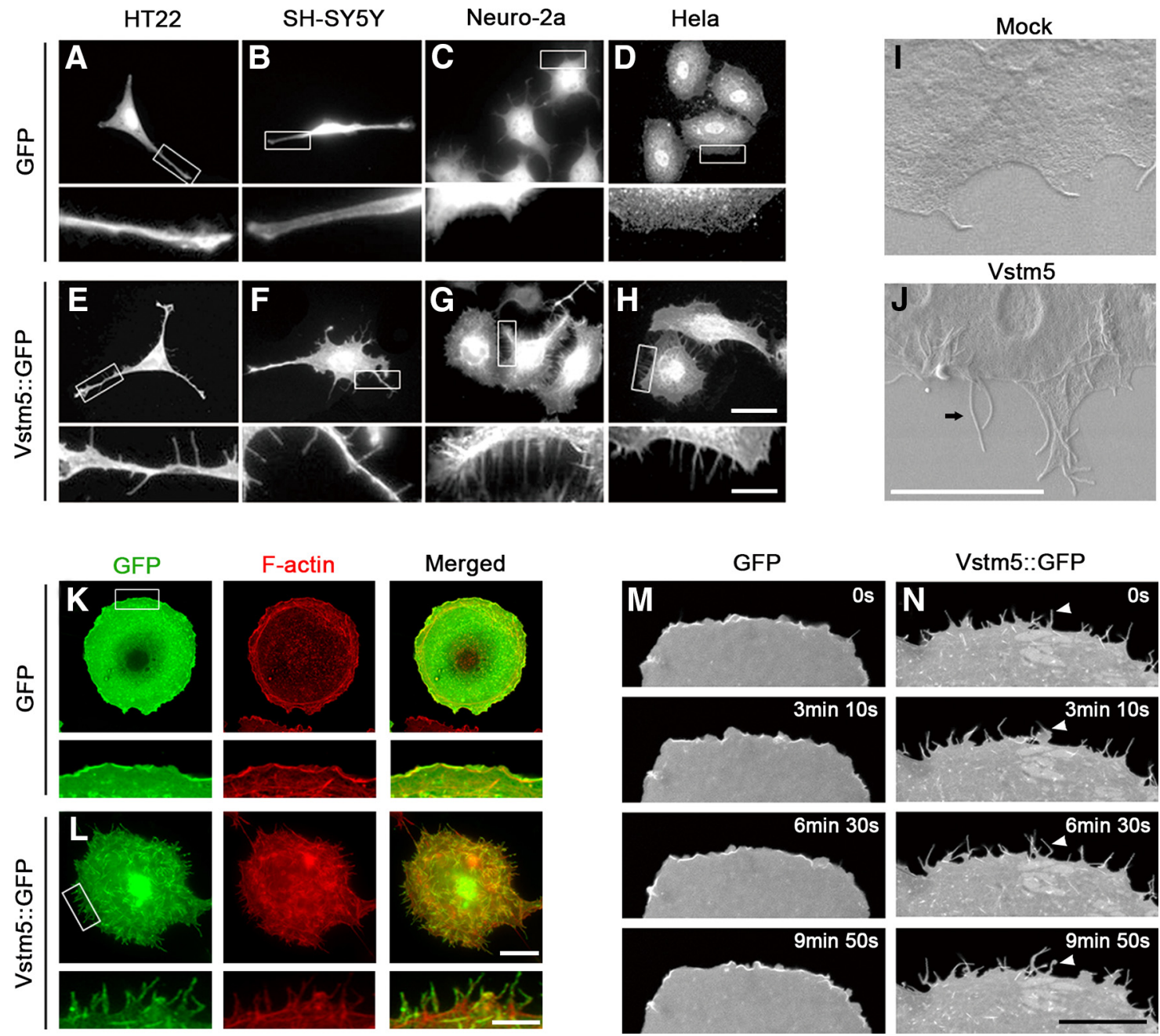

Figure 3. Vstm5 induces filopodia in various cell lines. $\boldsymbol{A}-\boldsymbol{H}$, Immunocytochemistry of GFP-expressing (A-D) and Vstm5::GFP-expressing ( $\boldsymbol{E}-\boldsymbol{H})$ cells (HT22, SH-SY5Y, Neuro-2a, and HeLa) stained with anti-GFP antibody. $\boldsymbol{I}, \boldsymbol{J}$, Scanning electron microscope micrograph of control (Mock; $\boldsymbol{I}$ ) and Vstm5-expressing $\operatorname{COS-7}$ cells $(\boldsymbol{J})$. Arrow indicates a hair-like filopodia structure. $\boldsymbol{K}, \boldsymbol{L}$, GFP-expressing $(\boldsymbol{K})$ and Vstm5:::GFP-expressing ( $\boldsymbol{L}) \operatorname{COS}-7$ cells were stained with anti-GFP antibody (green) and phalloidin (red) and their resultant images were merged (yellow). $\boldsymbol{M}, \boldsymbol{N}$, Time-lapse confocal series of GFP-expressing $(\boldsymbol{M})$ and Vstm5::GFP-expressing $(\boldsymbol{N})$ COS-7 cells collected every $10 \mathrm{~s}$ for $10 \mathrm{~min}$. Arrowheads indicate dynamic changes in a single filopodium. The bottom images in each row are magnified views of the white rectangular areas in the respective upper images. Scale bars: (in $\boldsymbol{H}$ ) $\boldsymbol{A}-\boldsymbol{H}$, low, $20 \mu \mathrm{m}$; high, $5 \mu \mathrm{m}$; (in $\boldsymbol{J}) \boldsymbol{I}, \boldsymbol{J}, 5 \mu \mathrm{m}$; (in $\boldsymbol{L}) \boldsymbol{K}, \boldsymbol{L}$, low, $30 \mu \mathrm{m}$; high, 10 $\mu \mathrm{m} ;$ (in $N) M, N, 20 \mu \mathrm{m}$.

edges of the cell (Fig. 3J). These latter protrusions resembled membrane filopodia seen in other cells (Wood and Martin, 2002). Because conventional filopodial structures are tightly linked to parallel bundles of actin filaments (Mattila and Lappalainen, 2008; Hotulainen and Hoogenraad, 2010), F-actin was visualized by labeling the cells with phalloidin. It was evident that the filopodia induced by Vstm5::GFP expression were colocalized with F-actin (Fig. 3L). Expression of Vstm5 triggered highly dynamic membrane properties, with formation of numerous filopodia and ruffles (Fig. 3N, Movie 2), compared with control expression of GFP (Fig. 3M, Movie 1). These results demonstrate that Vstm5 is a novel membrane protein, inducing dynamic filopodial protrusions in the cell membrane.

\section{Vstm5 C-terminal region is important for}

\section{filopodia formation}

Because membrane proteins involved in filopodia formation interact with actin filaments via their cytosolic domains (Lin et al., 2007), we investigated whether the cytosolic C terminus of Vstm5 is critical for the induction of membrane protrusions. A series of deletion mutations were constructed based on the charge property of the amino acid residues in the $\mathrm{C}$ terminus (Fig. 4A). Wild-type (WT) and mutant Vstm5 individually tagged with GFP were transfected into COS-7 cells and stained with an anti-GFP antibody (green) and phalloidin (red) (Fig. $4 B-F)$. Quantitative analysis showed that the density of filopodia increased by 7.2-fold in cells expressing Vstm5::GFP $(4.49 \pm 0.26 / 20 \mu \mathrm{m})$ compared with that in GFP-transfected control cells $(0.63 \pm 0.13 / 20 \mu \mathrm{m}$; Fig. $4 B, C, G)$. In cells expressing a mutant Vstm5 lacking the entire $C$ terminus (Vstm5-CTD) or a mutant missing the $\mathrm{N}$-terminal 17 residues of the C terminus (Vstm5-C[N $\Delta 17]$ ), filopodia density was greatly reduced $(0.93 \pm 0.18 / 20 \mu \mathrm{m}$ for Vstm5-CTD and $1.83 \pm 0.22 / 20 \mu \mathrm{m}$ for Vstm5-C $[\mathrm{N} \Delta 17])$ compared with that of WT cells (Fig. $4 D, F, G$ ). The Vstm5-CTD mutant failed to target to the cell surface and no longer colocalized with F-actin (Fig. 4D). However, a mutant lacking the C-terminal 14 residues of the $\mathrm{C}$ terminus, Vstm5-C $(\mathrm{C} \Delta 14)$ induced filopodia as strongly as WT Vstm5, suggesting the importance of the positive charges clustered in the first half of the cytosolic $\mathrm{C}$ terminus (Fig. 4A,E,G). Despite the marked effects of WT and mutant Vstm5 on filopodial density, no significant difference 


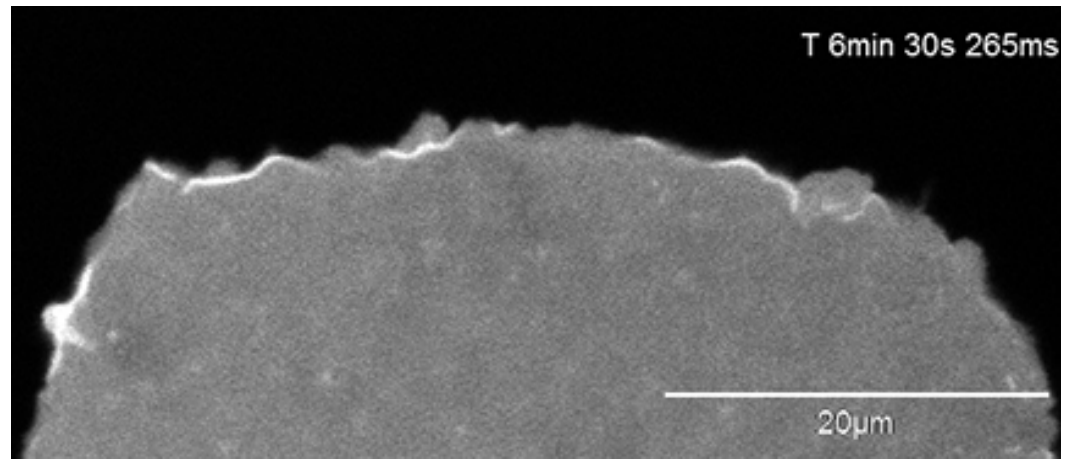

Movie 1. Time-lapse confocal series of GFP-expressing cells. Confocal live images were acquired every $10 \mathrm{~s}$ for $10 \mathrm{~min}$. Scale bar, $20 \mu \mathrm{m}$. (Fig. 3M).

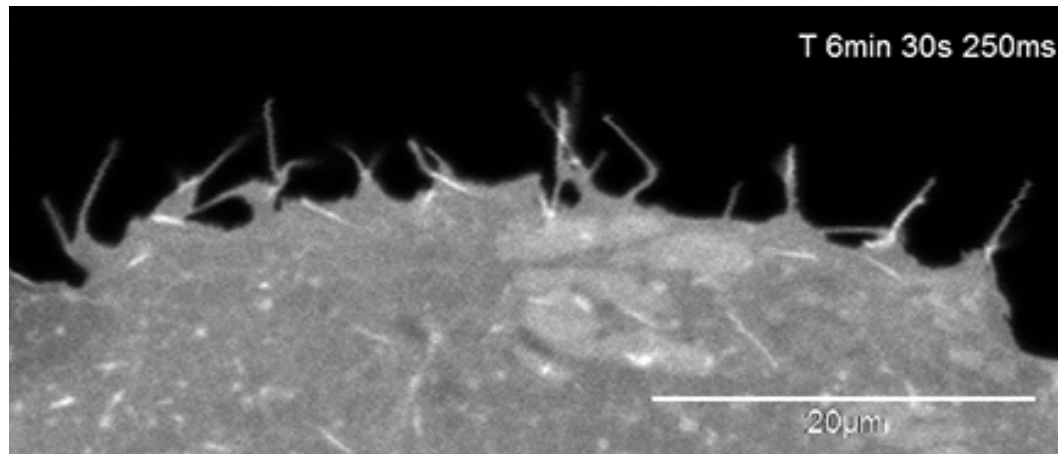

Movie 2. Time-lapse confocal series of Vstm5::GFP-expressing COS-7 cells. Confocal live images were acquired every $10 \mathrm{~s}$ for $10 \mathrm{~min}$. Scale bar, $20 \mu \mathrm{m}$. (Fig. 3N).

was observed in the length of filopodia (Fig. $4 H$ ). These results indicate that the juxtamembrane region of the cytosolic $\mathrm{C}$ terminus (CNQCAYKFQRKRRYKLK), and possibly the positively charged residues, are critical for Vstm5-induced filopodia.

In neuronal dendrites, membranous structural changes are essential for the formation of neuronal processes and dendritic filopodia are responsible for initiating synaptic contact with nearby axons in developing neurons (Ziv and Smith, 1996). To examine the role of Vstm 5 on dendritic filopodia induction, cultured mouse hippocampal neurons were transfected with control GFP, Vstm5::GFP, and Vstm5-C(N $\Delta 17):: G F P$ at DIV5 and imaged at DIV8 (Fig. 4I-K). In neurons expressing WT Vstm5::GFP, we observed a substantial increase in dendritic filopodia $(0.49 \pm 0.04 / \mu \mathrm{m})$ compared with that in GFP control $(0.26 \pm 0.04 / \mu \mathrm{m}$; Fig. $4 I, J, L)$. In addition, the density of dendritic filopodia $(0.14 \pm 0.04 / \mu \mathrm{m})$ was significantly decreased in neurons expressing Vstm5-C(N $\Delta 17)::$ GFP compared with that in the GFP control and Vstm5-overexpressing neurons (Fig. 4I$L$ ), suggesting a dominant-negative effect for this mutant Vstm5. There was no significant difference in the average length of dendritic filopodia between these neurons (Fig. 4M). These results are consistent with the aforementioned findings in COS-7 cell lines and further indicate a functional significance of Vstm5 in the formation of neuronal dendritic filopodia and the importance of the positively charged residues in the juxtamembrane region of the $\mathrm{C}$ terminus.

\section{Vstm5 regulates dendritic filopodia formation via a Cdc42-dependent pathway}

Rho GTPases (RHO1, CDC42, RAC1) are key regulators of cytoskeletal dynamics and affect various cellular processes, including cell polarity and migration (Heasman and Ridley, 2008). Cdc42 and Rac1 are the bestcharacterized members of the Rho/Rac GTPase families and are involved in the regulation of rearrangements of actin and membrane. We generated shRNAs against Cdc42 and Racl (Fig. 4T) and checked the effects of Cdc42 and Rac1 knock-down on neuronal morphology. DIV3 neurons were transfected individually with shScramble, shCdc42, or shRacl (Fig. $4 N-P$ ) or cotransfected with Vstm5::1D4 and shScramble, shCdc42, or shRacl (Fig. 4Q-S) and the number of dendritic filopodia at DIV7 was analyzed (Fig. $4 U$ ). In the single-transfected neurons, the number of filopodia was significantly different between shCdc42and shRacl-transfected neurons and shScramble-transfected neurons $(0.22 \pm$ $0.01 / \mu \mathrm{m}$ for $\mathrm{shCdc} 42,0.35 \pm 0.03 / \mu \mathrm{m}$ for shRac1, and $0.28 \pm 0.02 / \mu \mathrm{m}$ for shScramble). Vstm5::1D4 cotransfected with shScramble significantly increased filopodia density $(0.44 \pm 0.03 / \mu \mathrm{m})$ compared with shScramble single-transfected neurons. However, the number of dendritic filopodia was not significantly different between Cdc42-knock-down neurons and neurons cotransfected with Vstm5::1D4 and shCdc42 (0.22 \pm $0.01 / \mu \mathrm{m}$ for $\operatorname{shCdc} 42$ and $0.23 \pm 0.02 / \mu \mathrm{m}$ for shCdc42 plus Vstm5::1D4). These results suggest that Vstm5-induced dendritic filopodia formation involves the Cdc42 pathway, a well known cellular pathway involved in membrane dynamics change.

Next, to determine whether Cdc42 or Racl is involved in Vstm5-induced membrane, we performed the Cdc42/Rac1 activation assay (Fig. $4 V-X$ ). Upon Vstm 5 overexpression, the levels of activated Cdc42 and Racl were significantly increased. In contrast, in the Vstm5-C(N $\Delta 17)$ mutant, the levels of activated Cdc42 and Rac1 were lower than in the WT. Therefore, these results strongly suggest that $\mathrm{Cdc} 42$ is required for Vstm5-induced changes in membrane dynamics and the juxa-transmembrane region of Vstm5 may activate Cdc42.

\section{Vstm5 regulates dendritic filopodia and dendrites in mouse hippocampal neurons}

To determine the functional role of Vstm5 in neurons, we investigated the effects of an shRNA against endogenous Vstm5 in mouse hippocampal neurons. The shRNA against Vstm5 (shVstm5) and a scrambled control shRNA (shScramble) were separately constructed in IRES-GFP containing pCAG-mir30 vectors. Initially, we validated the efficacy of the shRNA in HEK 293 cells by cotransfecting with nontagged Vstm 5 because 


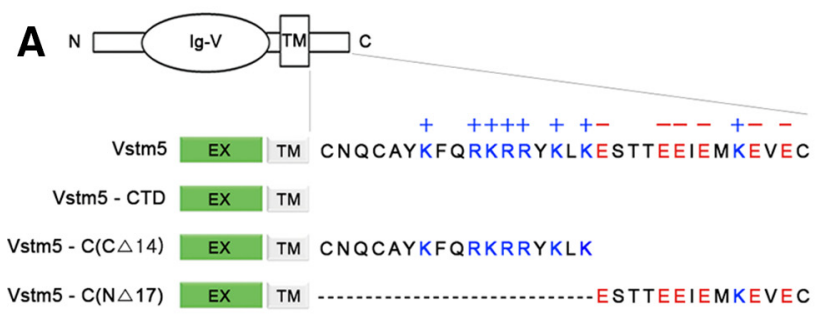

G
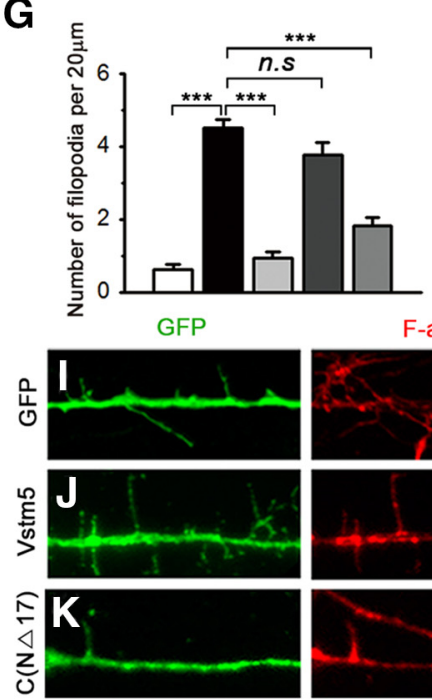

shScramble

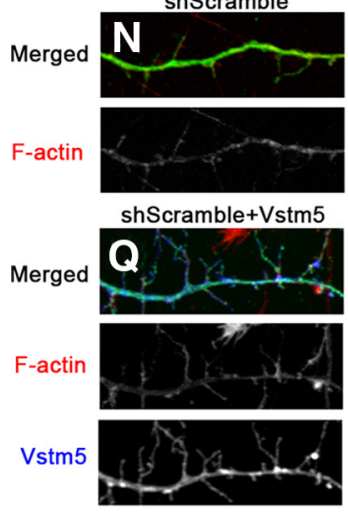

$\mathbf{T}$

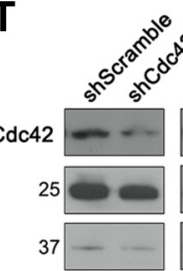

H

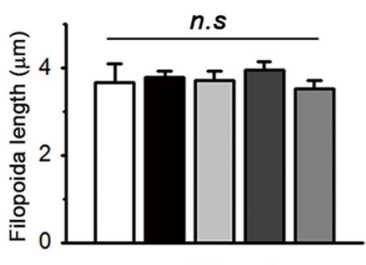

Merged
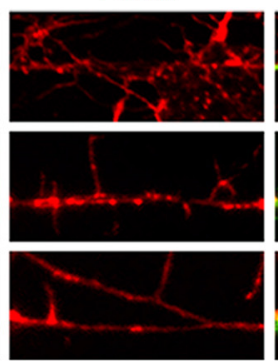

$\operatorname{shCdc42}$

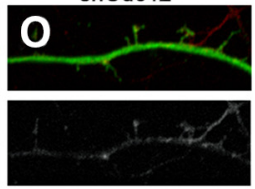

$\operatorname{shCdc} 42+$ Vstm5
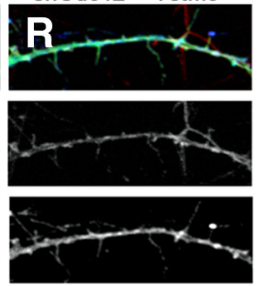

$\mathbf{U}$

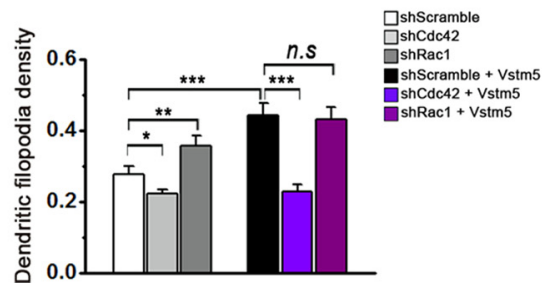

L

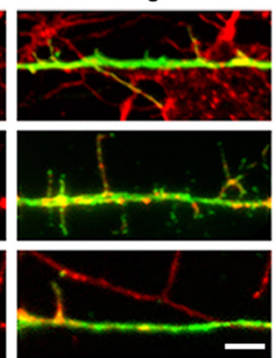

shRac1
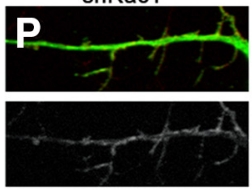

shRac1 + Vstm5

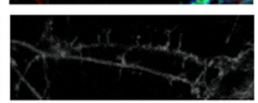

V
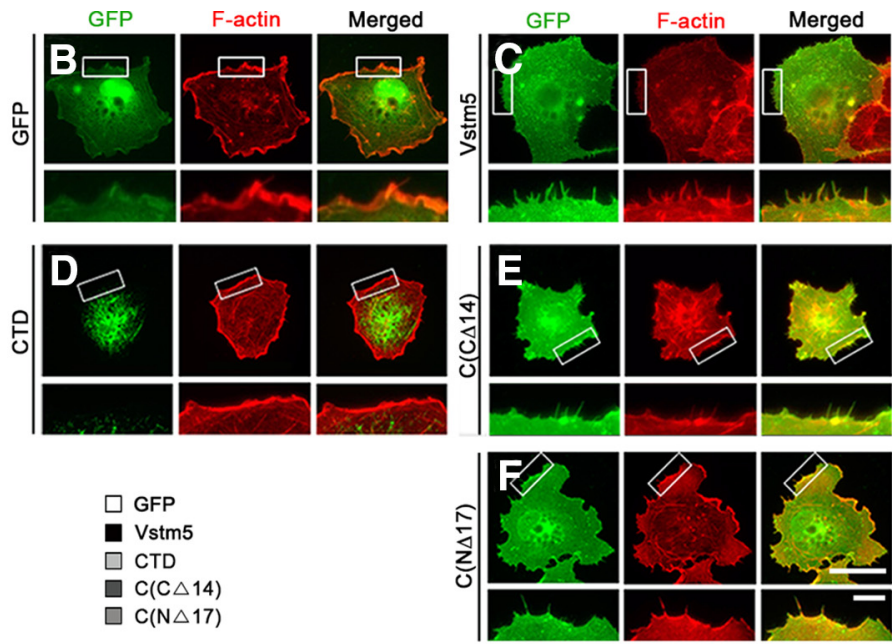

$\square$ GFP

口 Vstm5

$\square$ CTD

$\square \mathrm{C}(\mathrm{C} \triangle 14)$

$\square \mathrm{C}(\mathrm{N} \triangle 17)$
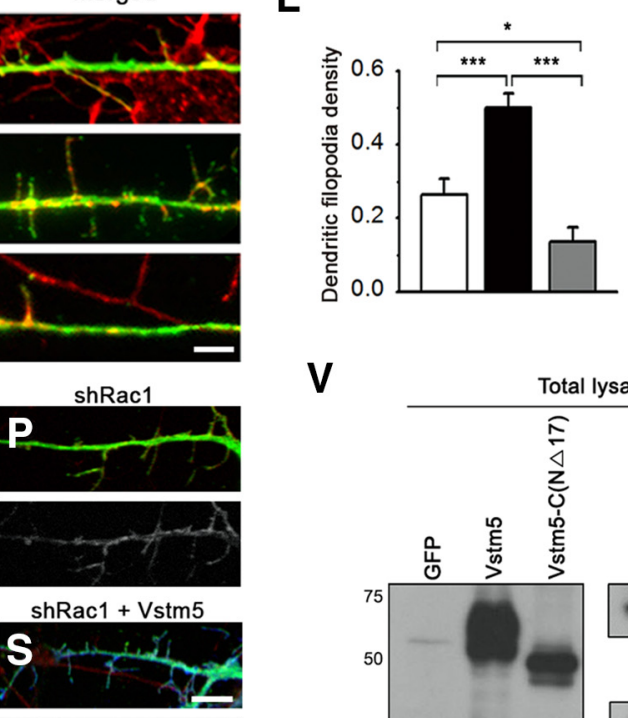

M

$\square$ Control

- Vstm5 $\square \mathrm{C}(\mathrm{N} \triangle 17)$$$
\text { 흠 }
$$
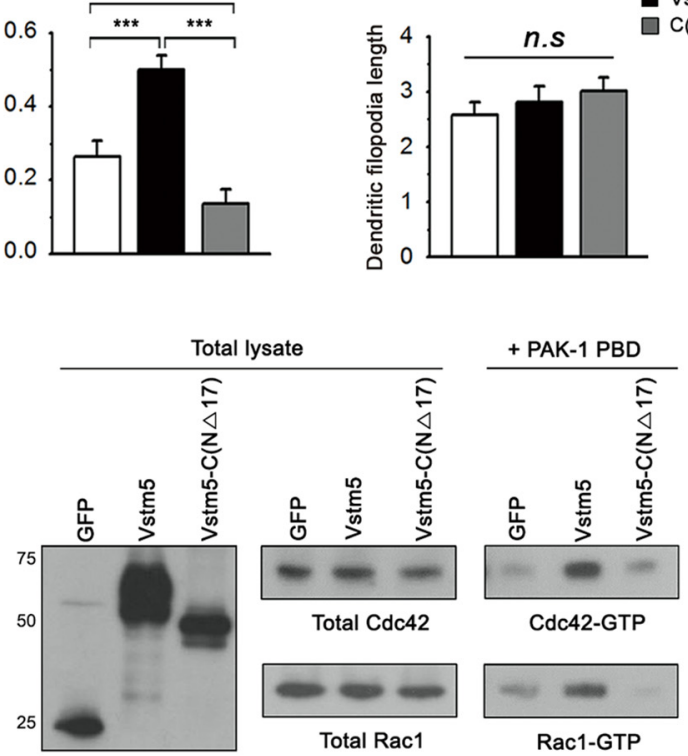

W

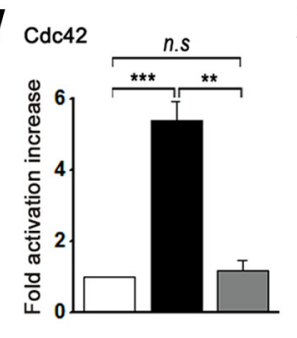

Figure 4. The juxtamembrane ( terminus region of Vstm 5 is important for Cdc42-dependent filopodia induction. $\boldsymbol{A}$, Schematic diagram of C-terminal mutant Vstm 5 constructs. C-terminal mutants of Vstm 5 constructs were generated based on the property of charged residues. Color and symbol indicate positively ( + , blue) and negatively ( - , red) charged residues. $\boldsymbol{B}-\boldsymbol{F}$, GFP-, Vstm5-, and C-terminal mutant-transfected COS-7 cells stained with an anti-GFP antibody (green) or phalloidin (red). $\mathbf{G}, \boldsymbol{H}$, Number of filopodia per $20 \mu \mathrm{\mu m}(\boldsymbol{G})$ and length of filopodia $(\boldsymbol{H})$ in transfected COS-7 cells from $\boldsymbol{B}-\boldsymbol{F}$ (GFP, $n=20$; Vstm5, $n=31$; Vstm5-CTD, $n=20$; Vstm5-C(C $\Delta 14), n=20$; and Vstm5-C(N $\Delta 17), n=23)$. I- $\boldsymbol{K}$, Mouse hippocampal neurons (DIV5) transfected with control GFP $(\boldsymbol{I})$, Vstm5::GFP $(\boldsymbol{J})$, and Vstm5-C(N $\Delta 17):: G F P(\boldsymbol{K})$ and immunostained with GFP (green) and phalloidin (red) at DIV8. $\boldsymbol{L}, \boldsymbol{M}$, Density $(\boldsymbol{L})$ and length $(\boldsymbol{M})$ of dendritic filopodia per $\mu \mathrm{m}$ dendrite in

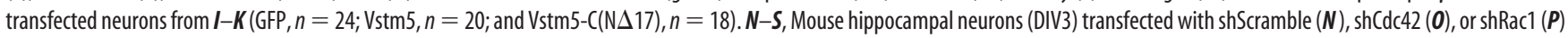
or cotransfected with Vstm5::1D4 and shScramble (Q), shCdc42 (R), or shRac1 (S) and immunostained with GFP (green), phalloidin (red) and 1D4 (blue) at DIV7. $\boldsymbol{T}$, Verification of knock-down of

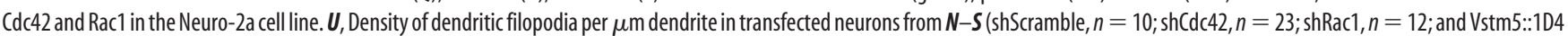
cotransfected with shScramble, $n=16$; shCdc42, $n=19 ;$ shRac1, $n=15)$. V, Cells were transfected with pEGFP or GFP fused to Vstm5 or Vstm5-C(N $\Delta 17)$. After 72 h, cells were lysed and incubated with PAK-1 RBD (p21 binding domain of PAK-1) to pull down active (dc42 and Rac1, respectively. Samples were then blotted with (dc42 and Rac1 antibodies. The middle panel gels are Western blots of total cell lysates, which were used as loading controls. $\boldsymbol{W}, \boldsymbol{X}$, Bands from the active $(\mathrm{d} c 42$ and active Rac 1 gels were quantified using ImageJ software and then normalized to the amount of total protein $\left(n=3\right.$; mean \pm SEM). Error bars indicate SEM. ${ }^{*} p<0.05,{ }^{* *} p<0.001$; ns, no statistical significance. The bottom images in each row are magnified views of the boxed areas in the respective upper images. Scale bars: (in $\boldsymbol{F}$ ) $\boldsymbol{B}-\boldsymbol{F}$, low, $35 \mu \mathrm{m}$; high, $5 \mu \mathrm{m}$; (in $\boldsymbol{K}) \boldsymbol{I}-\boldsymbol{K}, 5 \mu \mathrm{m}$; (in $\boldsymbol{S}) \boldsymbol{N}-\boldsymbol{S}, 5 \mu \mathrm{m}$. 

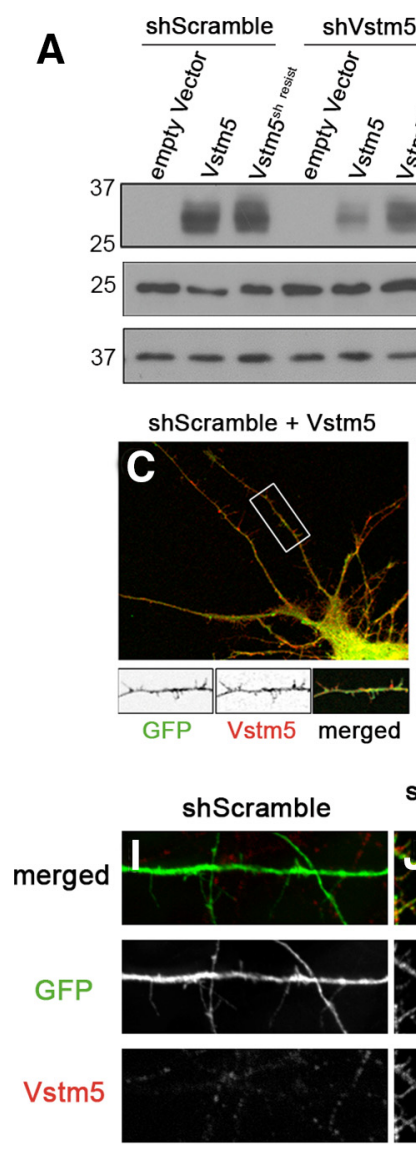

shScramble
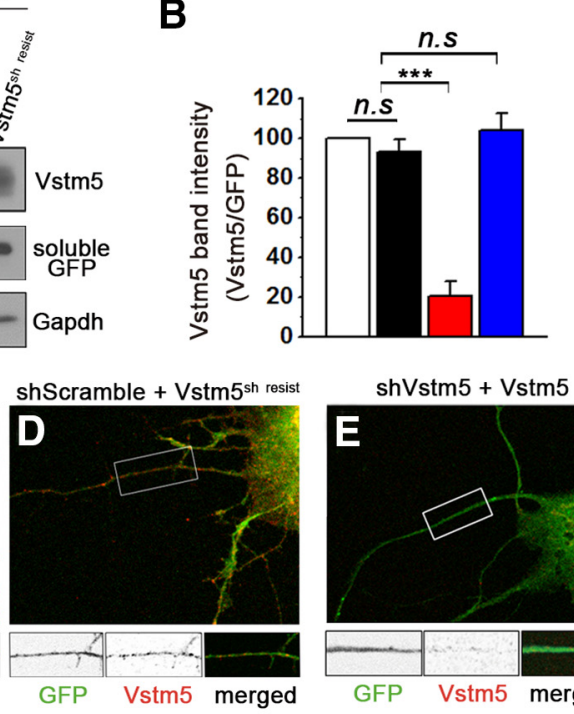

G
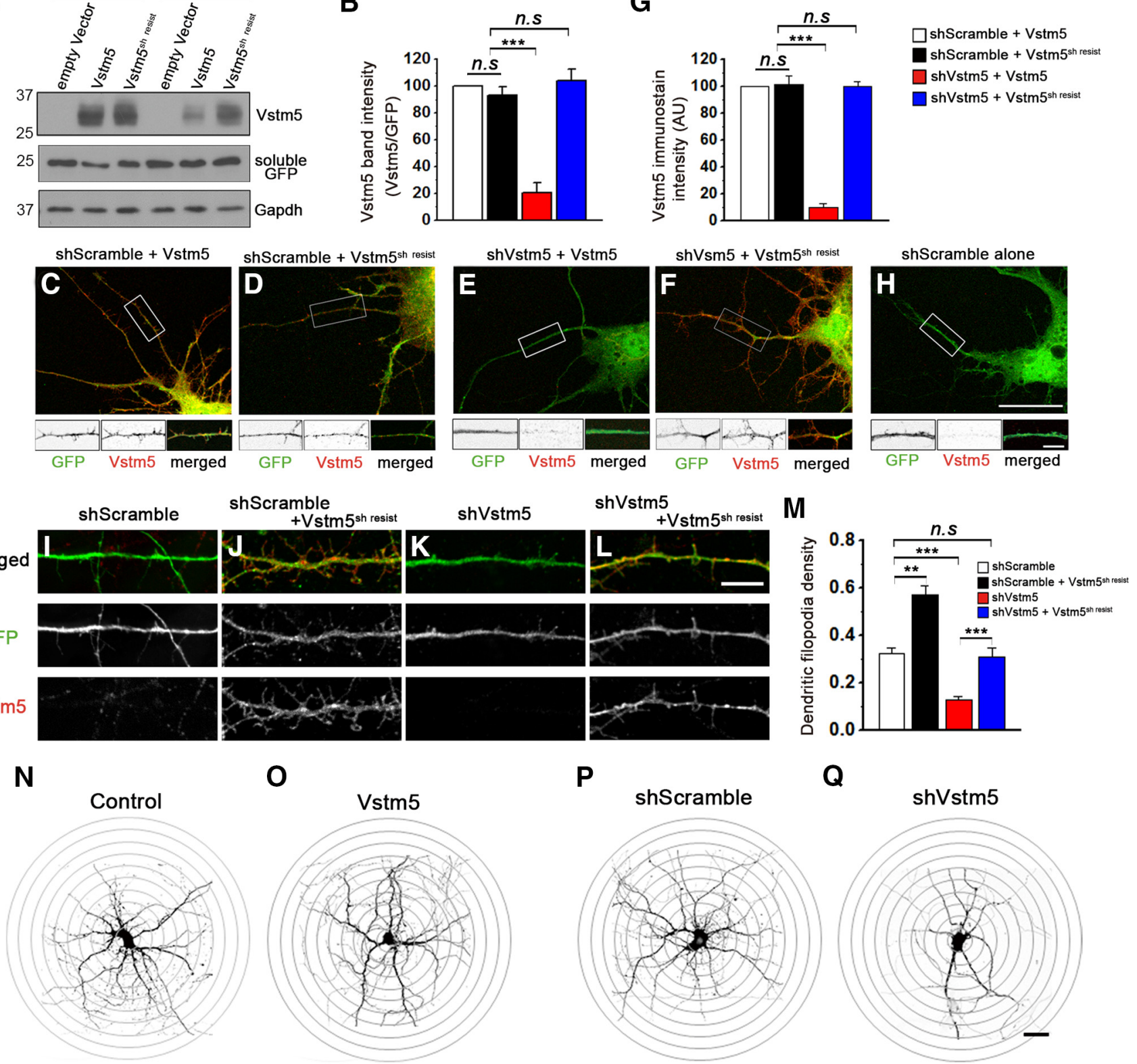

$\mathbf{P}$
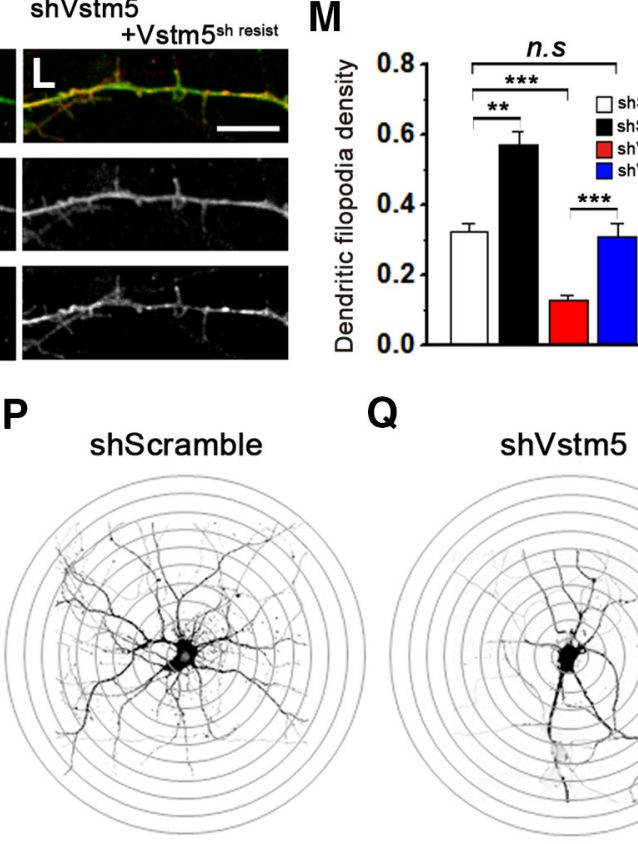

$\mathbf{R}$

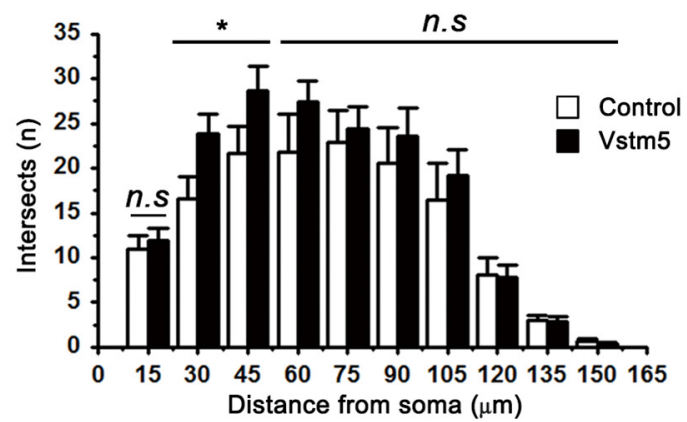

S

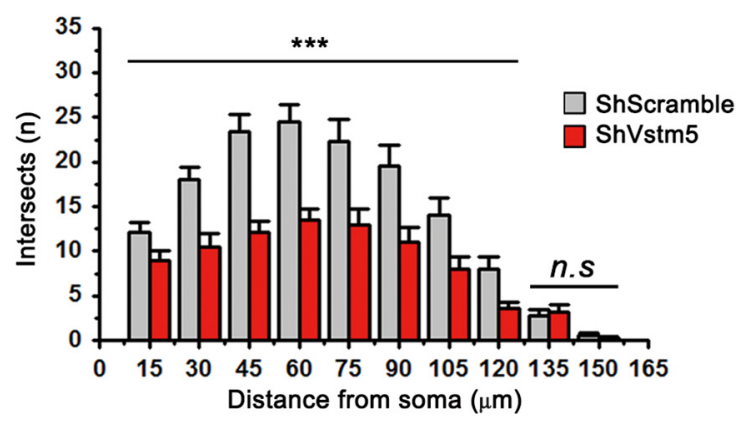

Figure 5. Vstm5 regulates dendritic filopodia density and dendritic complexity in neurons. A, Cells transfected with shScramble or shVstm5 and cotransfected with pcDNA3.1, Vstm5/pcDNA3.1, or Vstm $5^{\text {sh resist } / p C D N A 3.1 ~ w e r e ~ s u b j e c t e d ~ t o ~ W e s t e r n ~ b l o t t i n g ~ a n a l y s i s ~ u s i n g ~ a n t i-V s t m 5, ~ a n t i-G F P, ~ a n d ~ a n t i-m G A P D H ~ a n t i b o d i e s . ~ B, ~ Q u a n t i f i c a t i o n ~ o f ~ r e l a t i v e ~ b a n d ~ i n t e n s i t i e s . ~ L e v e l s ~ o f ~ V s t m ~} 5$ in lanes 2, 3 and 5, 6 were normalized to GFP expression and are displayed as histograms. $\mathbf{C}-\boldsymbol{F}, \boldsymbol{H}$, Transfected neurons were immunostained with anti-GFP (green) and anti-Vstm5 (red) antibodies. Merged confocal panels show neurons overexpressing shScramble alone $(\boldsymbol{H})$ and shScramble plus untagged Vstm5 $(\boldsymbol{C})$ or Vstm5 ${ }^{\text {sh resist }}(\boldsymbol{D})$ and shVstm5 plus Vstm5 $(\boldsymbol{E})$ or Vstm5 ${ }^{\text {sh resist }}(\boldsymbol{F})$. A high-magnification view of the region enclosed by the rectangle is shown below each image. $\mathbf{G}$, Vstm5 immunofluorescence intensity (arbitrary units, $\mathrm{AU}$ ) in dendrites was imaged as in $\boldsymbol{C}-\boldsymbol{F}(n=$ 4 neurons each). $\boldsymbol{I}-\boldsymbol{L}$, shScramble- $(\boldsymbol{I})$ and shVstm5- $(\boldsymbol{K})$ and Vstm 5 sh resist cotransfected shScramble-expressing $(\boldsymbol{J})$ and shVstm5-expressing $(\boldsymbol{L})$ neurons (DIV8) were immunostained with anti-GFP ( green), phalloidin (red) and 1D4 (blue), and the images were merged. $\boldsymbol{M}$, Effect of shVstm5 on the density of dendritic filopodia in transfected neuronal dendrites from $\boldsymbol{I}-\boldsymbol{L}$ (shScramble, $n=15$; shVstm5, $n=17$; Vstm5 ${ }^{\text {sh }}$ resist plus shScramble, $n=17$; shVstm5, $n=17$ ). $\mathbf{N}-\mathbf{Q}$, Inverted images of each construct-expressing neuron (Figure legend continues.) 
the best available antibody against Vstm 5 detected Vstm 5 only in Vstm5-overexpressing cells and was not suitable for detecting endogenous protein (Fig. $5 \mathrm{H}$ ). Based on quantitative immunoblotting results, the shRNA against Vstm5 suppressed the expression level of Vstm 5 to $20.5 \pm 7.3 \%$ of that in the vector-transfected control. This decrease was rescued by coexpression of the Vstm $5^{\text {sh resist }}$ construct (Fig. $5 A, B$ ). When tested on cultured hippocampal neurons transfected with WT Vstm5, shVstm5 decreased the intensity of exogenous Vstm5 expression to $10.1 \pm 2.9 \%$ of that observed with shScramble and Vstm $5^{\text {sh resist }}$ rescued the decrease in intensity to $99.8 \pm 3.9 \%$ (Fig. 5C-G).

To determine the effects of knocking down endogenous Vstm5 on dendritic filopodia formation, we transfected shScramble or shVstm5 into mouse hippocampal neurons at DIV5 and immunostained for GFP and phalloidin at DIV8 (Fig. 5I,K). The density of dendritic filopodia was significantly lower in Vstm5-knock-down neurons $(0.13 \pm 0.02 / \mu \mathrm{m})$ compared with that in shScrambleexpressing neurons $(0.33 \pm 0.02 / \mu \mathrm{m}$; Fig. $5 M)$. This effect was rescued by coexpression of knock-down-resistant Vstm5 (shVstm5 + Vstm5 ${ }^{\text {sh }}$ resist, $0.31 \pm 0.04 / \mu \mathrm{m}$; Fig. $5 L, M$ ). Overexpression of $\mathrm{Vstm} 5^{\text {sh resist }}$ in the presence of endogenous protein increased filopodia density (shScramble + Vstm $5^{\text {sh resist }}, 0.57 \pm 0.04 / \mu \mathrm{m}$; Fig. 5 $J, M)$. These results also support Vstm5 regulation of the formation of dendritic filopodia, at least in mouse hippocampal neurons.

Proteins inducing dendritic protrusions are also associated with dendrite formation (Alfonso et al., 2005; Crespi et al., 2012). We thus examined the effects of Vstm 5 on dendritic complexity in mouse hippocampal neurons using the Sholl analysis at DIV14. In this analysis, the number of dendritic intersections crossing each $15 \mu \mathrm{m}$ radius ring was counted progressively more distal from the soma to $150 \mu \mathrm{m}$. Neurons of both experimental pairs (GFP control vs Vstm5, and shScramble vs shVstm5) showed statistically significant differences in their dendritic complexities (Fig. 5N-Q). In neurons expressing exogenous Vstm5, the dendrites were significantly more complex in the region between 30 and $60 \mu \mathrm{m}$ compared with control dendrites (Fig. 5N,O,R). More profound effects were evident, however, in Vstm5-knockdown neurons. The dendritic complexity was significantly decreased in a broader region from 15 to $120 \mu \mathrm{m}$. At the $45 \mu \mathrm{m}$ radius, the complexity of neuronal dendrites was decreased by $50 \%$ after knocking down Vstm5, from $23.4 \pm 1.9$ in shScramble to $12.2 \pm 1.1$ in shVstm5 (Fig. $5 P, Q, S$ ). These results reinforce the idea that Vstm 5 regulates neuronal morphology by developing new dendrites and inducing additional dendritic filopodia.

\section{Vstm 5 regulates dendritic spine morphology and density}

When expressed in neurons, Vstm5 is found in dendritic and axonal membranes in an uneven manner (Fig. 6A-H). However, Vstm 5 induces membrane protrusions only in dendritic regions that can be visualized with microtubule-associated protein 2 (MAP2) antibodies (Fig. 6A-D), not in axonal membranes costained against neurofilament (Fig. $6 E-H)$. These observations

\section{$\leftarrow$}

(Figure legend continued.) (DIV14) merged with progressively increasing (5 to $150 \mu \mathrm{m}$ ) radii. $R, S$, Quantification of the number of dendritic intersections crossing each $15 \mu \mathrm{m}$ radius ring in control GFP-expressing $(\boldsymbol{N})$, Vstm5-expressing $(\boldsymbol{O})$, and shScramble-expressing $(\boldsymbol{P})$ and shVstm5-expressing ( $Q$ ) neurons (GFP, $n=20$; Vstm5, $n=25$; shScramble, $n=23$; and shVstm5, $n=21$ ). Error bars indicate SEM. ${ }^{*} p<0.05,{ }^{* *} p<0.01,{ }^{* * *} p<0.001 ; \mathrm{ns}, \mathrm{no}$ statistical significance. Scale bars: (in $\boldsymbol{H}) \mathbf{C}-\boldsymbol{F}, \boldsymbol{H}$, low, $20 \mu \mathrm{m}$; high, $5 \mu \mathrm{m}$; (in $\boldsymbol{L}) \boldsymbol{I}-\boldsymbol{L}, 5 \mu \mathrm{m}$. (in Q) $\mathbf{N}-\mathbf{Q}, 30 \mu \mathrm{m}$. suggest that Vstm5 may play a role in dendritic differentiation and synapse targeting.

Therefore, we carefully examined the morphological changes of dendritic spines induced by Vstm5. Dendritic spines can be categorized in accordance with their morphology (Harris et al., 1992; Pak et al., 2001). Mushroom spines are protrusions with a globular head and short neck, thin spines are filopodia-like protrusions with a small spine head, branched spines are branched thin or mushroom spines, and stubby spines are short protrusions without a discernible neck (Pak et al., 2001). Mouse hippocampal neurons were transfected with Vstm5 at DIV10 and fixed at DIV21 for quantitative analyses of spine density and morphology (Fig. 6I,J). Both total spine density and length were significantly increased in Vstm5-expressing neurons (0.58 \pm $0.04 / \mu \mathrm{m}$ and $1.78 \pm 0.08 \mu \mathrm{m}$, respectively) compared with those in control neurons $(0.34 \pm 0.02 / \mu \mathrm{m}$ and $1.50 \pm 0.10 \mu \mathrm{m}$; Fig. $6 K, M)$, whereas spine head width was decreased from $0.66 \pm$ $0.02 \mu \mathrm{m}$ in control to $0.52 \pm 0.01 \mu \mathrm{m}$ in Vstm5-transfected neurons (Fig. 6L). For the four spine categories, Vstm5 expression increased the densities of thin, stubby, and branched spines without significantly altering the density of mushroom spines (Fig. $6 N$ ). The density of branched spines, rarely seen in controls $(0.01 \pm 0.002 / \mu \mathrm{m})$, was increased 8-fold in Vstm5-transfected neurons. These results demonstrate that Vstm 5 affects, not only the number of filopodia-like dendritic spines, but also the overall morphology of dendritic spines at a later stage in development.

We then investigated the functional effects of endogenous Vstm5 on the filopodia-to-spine transition using shScramble and shVstm5 (Fig. 6O,P). In Vstm5-knock-down neurons, spine density was markedly decreased $(0.26 \pm 0.02 / \mu \mathrm{m})$ compared with that in the shScramble control $(0.40 \pm 0.04 / \mu \mathrm{m}$; Fig. $6 Q)$, without significant differences in spine head width or spine length (Fig. 6R,S). The proportion of mature dendritic spines was reduced, mainly due to a decrease in the number of mushroom spines, whereas that of stubby spines was increased in the Vstm5-knock-down neurons; thin and branched spines were unaffected (Fig. 6T). These observations further demonstrate that Vstm5 contributes to the regulation of dendritic spine density and morphology.

\section{Vstm5 induces synapse remodeling and actin reorganization in hippocampal neurons}

Morphological plasticity of dendritic spines correlates with subcellular distribution of the molecular contents and organelles within the spines and these changes contribute to the functional and structural plasticity of synapses (Sala et al., 2001). To assess the relationship between Vstm5-induced morphological changes in spines and synapse formation in the late stages of neuron development, we doubly stained neurons at DIV21 using presynaptic and postsynaptic markers (Fig. $7 A-D)$. When the colocalized puncta of postsynaptic density-95 (PSD-95; postsynaptic marker) and synaptophysin-1 (presynaptic marker) on the spines were counted, synapse density was significantly higher in Vstm5overexpressing neurons than in control neurons (control, $0.35 \pm$ $0.03 / \mu \mathrm{m}$; Vstm5, $0.83 \pm 0.06 / \mu \mathrm{m}$; Fig. $7 A, B, E)$. In contrast, in Vstm5-knock-down neurons, synaptic density was markedly reduced ( $\operatorname{shScramble,~} 0.41 \pm 0.03 / \mu \mathrm{m}$; $\operatorname{shVstm5}, 0.24 \pm 0.02 / \mu \mathrm{m}$; Fig. $7 C-E)$, suggesting that a limited supply of Vstm5 in the synapse may prevent proper maturation of excitatory synapses. Therefore, Vstm 5 may be required for maintaining the structures of dendritic spines and excitatory synapses in cultures.

The actin cytoskeleton determines directly the morphological plasticity of dendritic spines and filopodia (Matus et al., 1982; Matus et al., 2000). F-actin plays a critical role in sus- 


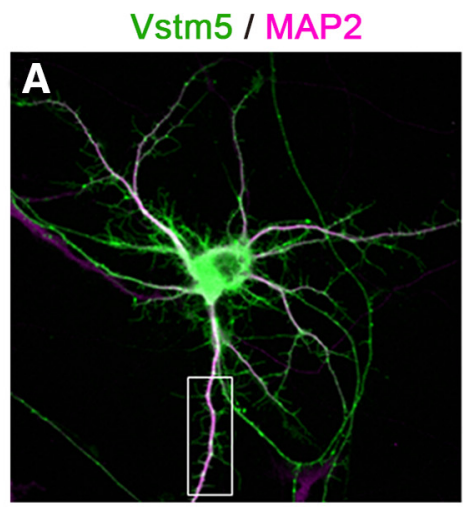

Control
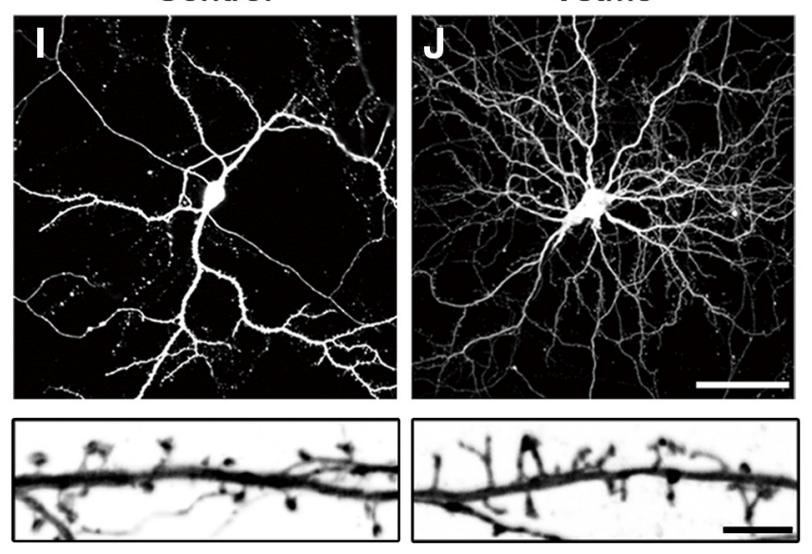

shScramble
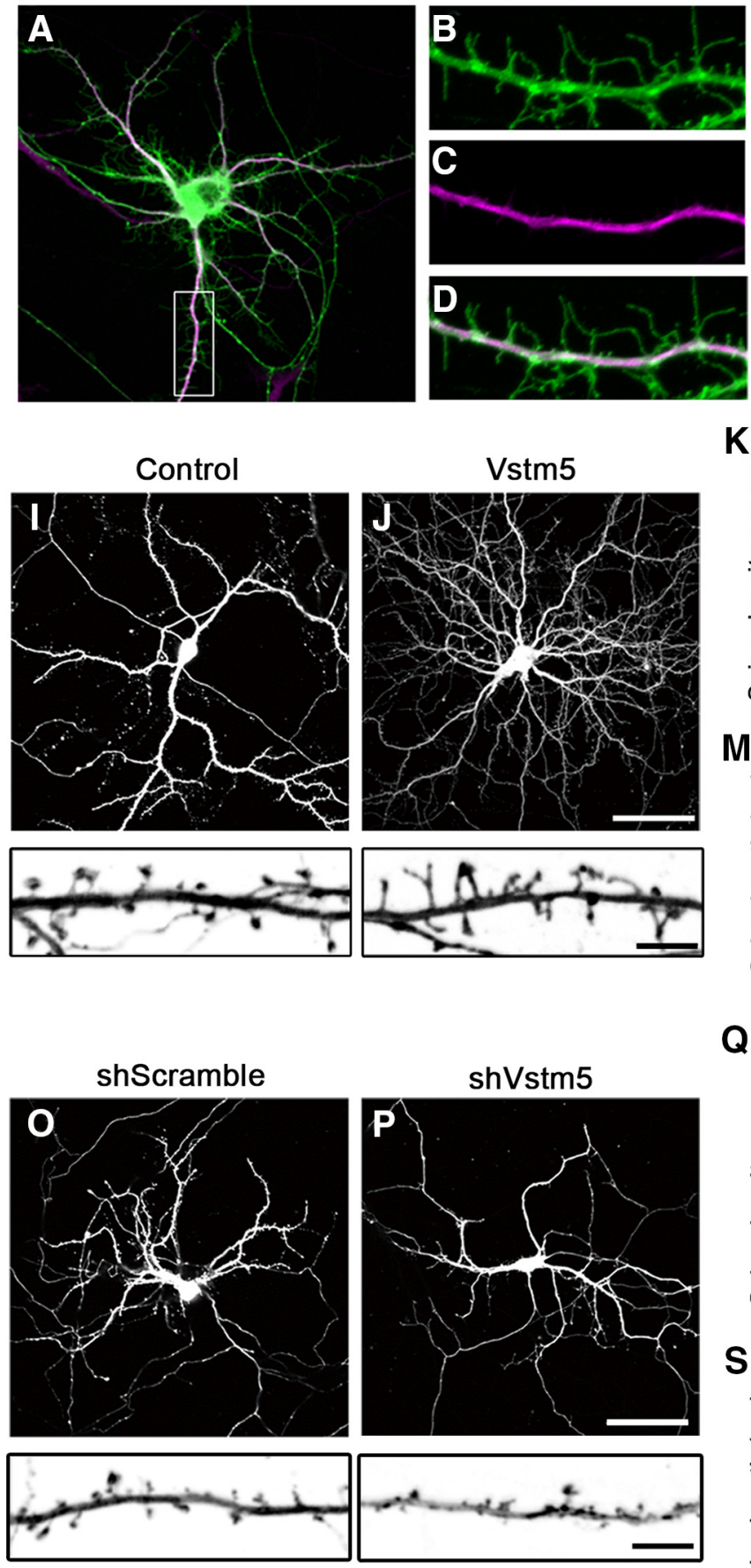

$\mathrm{K}$
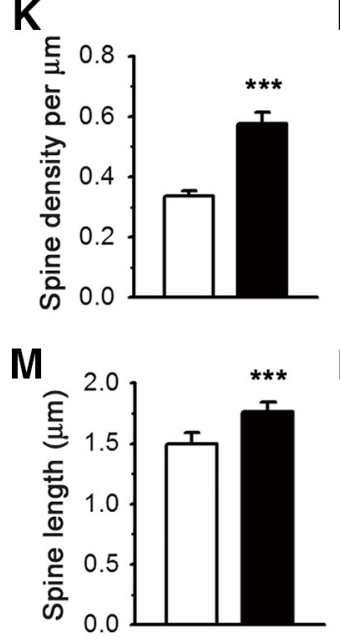

Q

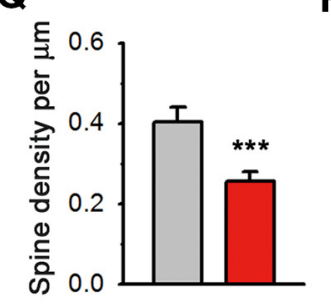

$\mathrm{S}$

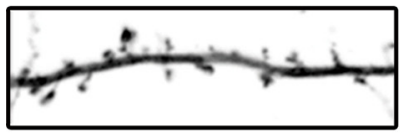

Vstm5 / Neurofilament
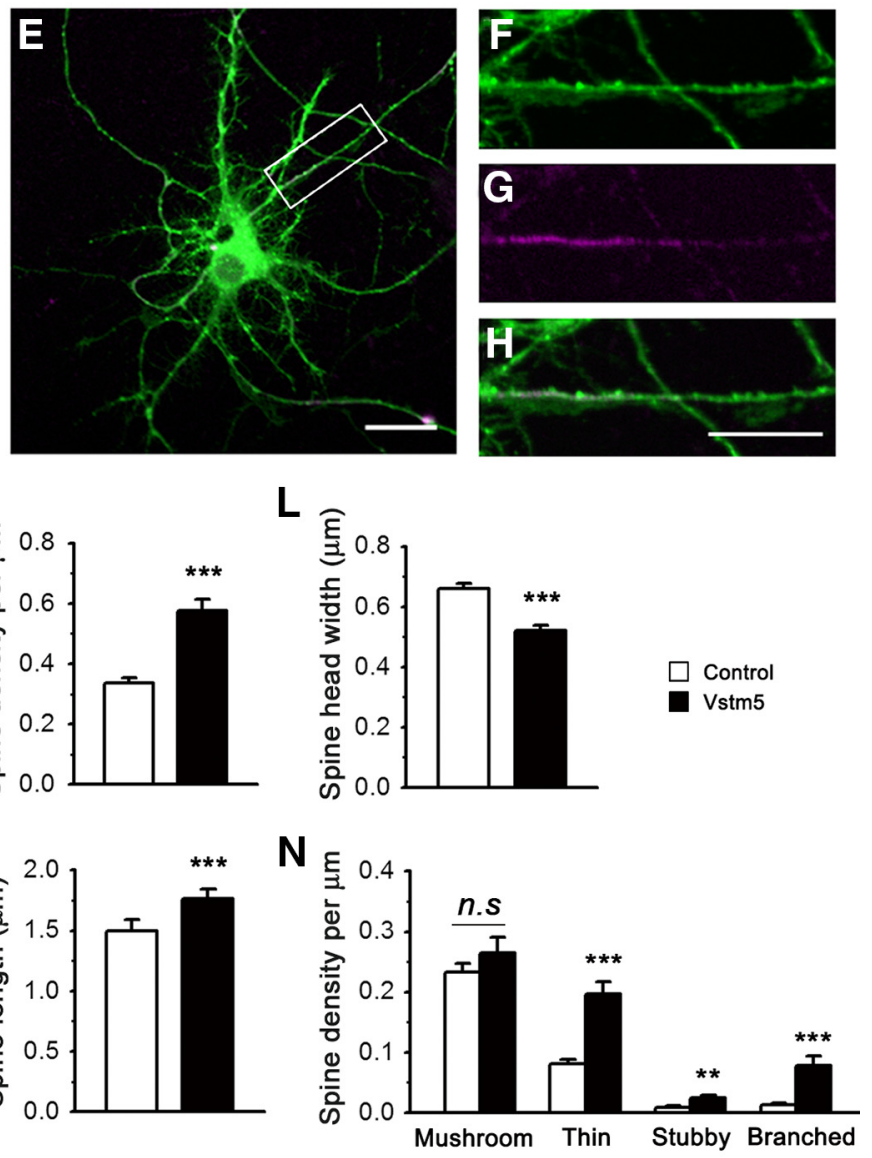

R
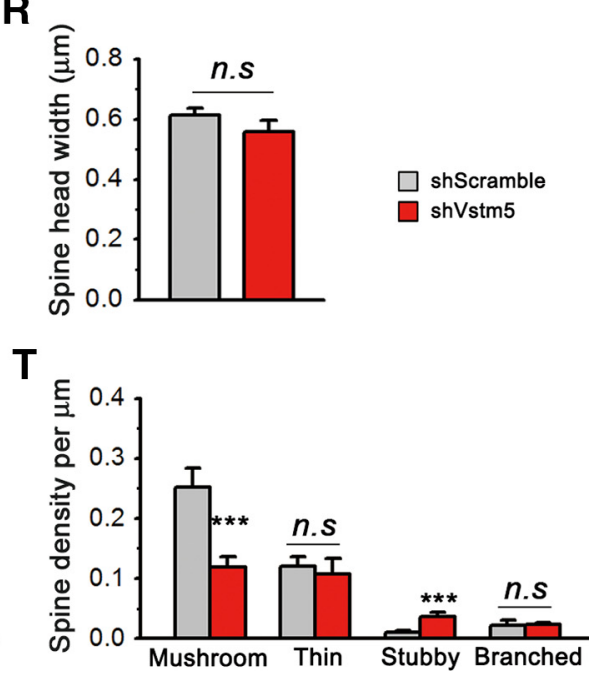

Figure 6. Vstm5 regulates dendritic spine morphology and density. $\boldsymbol{A}-\boldsymbol{H}$, Immunocytochemistry of Vstm5::GFP-expressing hippocampal neurons double stained with antibodies against GFP (green) and MAP2 (dendritic marker, purple; $\boldsymbol{A}-\boldsymbol{D}$ ) or neurofilament (axonal marker, purple; $\boldsymbol{E}-\boldsymbol{H}$ ) at DIV14. The right images are magnified views of the white rectangular areas in the respective images. $\boldsymbol{I}, \boldsymbol{J}, \mathbf{O}, \boldsymbol{P}$, Neurons were transfected at DIV10 after plating and imaged at DIV21 and immunostained with a GFP antibody. High-magnification images were inverted for clarity. $\boldsymbol{K}-\boldsymbol{M}, \mathbf{Q}-\boldsymbol{S}$, Histograms of spine density $(\boldsymbol{K}, \mathbf{Q})$, spine head width $(\boldsymbol{L}, \boldsymbol{R})$, and spine length $(\boldsymbol{M}, \boldsymbol{S})$ were plotted for transfected neurons. $\boldsymbol{N}, \boldsymbol{T}$, Dendritic spine density was quantified in four groups of spines classified by their shapes (Control, $n=9 ; \mathrm{Vstm} 5, n=11$; shScramble, $n=13$; and shVstm5, $n=16$ ). Error bars indicate SEM. ${ }^{* *} p<0.01,{ }^{* * *} p<0.001$; ns, no statistical significance. Scale bars: (in $E$ ) $A$, $\boldsymbol{E}, 20 \mu \mathrm{m}$; (in $\boldsymbol{H}) \boldsymbol{B}-\boldsymbol{D}, \boldsymbol{F}-\boldsymbol{H}, 10 \mu \mathrm{m}$; (in $\boldsymbol{J}, \boldsymbol{P}) \boldsymbol{I}, \boldsymbol{J}, \mathbf{O}, \boldsymbol{P}$, low, $50 \mu \mathrm{m}$; high, $5 \mu \mathrm{m}$.

taining motility during synaptic development and plasticity (Pilo Boyl and Witke, 2014); it is particularly concentrated in dendritic spine heads and forms a complex network to support dendritic spine structure in later stages of neuronal development (Matus et al., 1982). We observed that the exogenous expression of Vstm5 induced F-actin rearrangement from the dendritic protrusions to the shaft (Fig. $7 F, G, J-L$ ). In addition, Vstm5 knock-down also significantly decreased the spine to shaft ratio of F-actin immunofluorescence intensity (Fig. $7 \mathrm{H}-$ $J)$. Because disruption of the actin cytoskeleton did not considerably alter the expression pattern of Vstm5 (Fig. $7 \mathrm{M}, N$ ), it remains to be determined how the expression level of Vstm5 


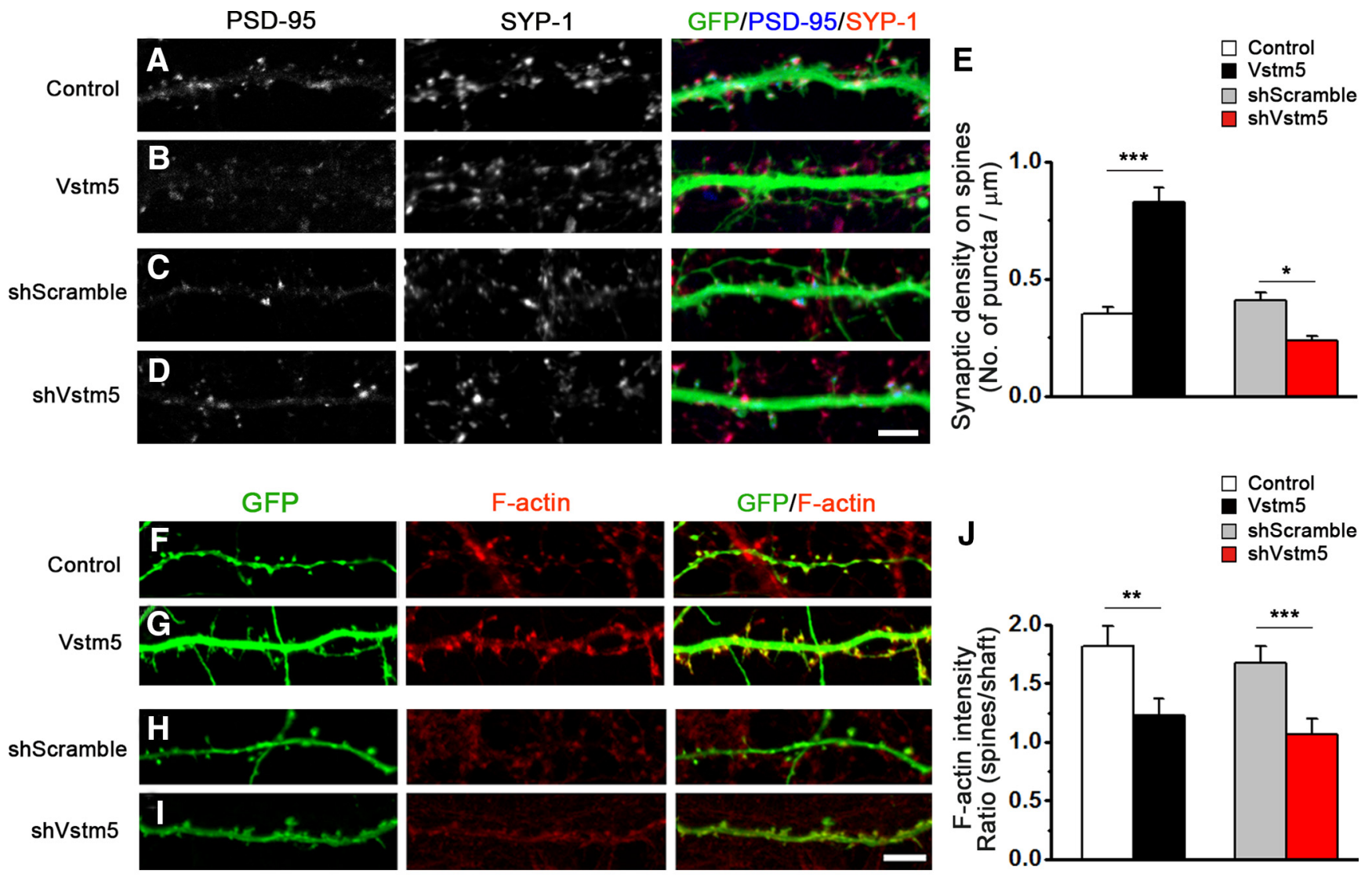

$\beta$-actin::GFP + pcDNA3.1
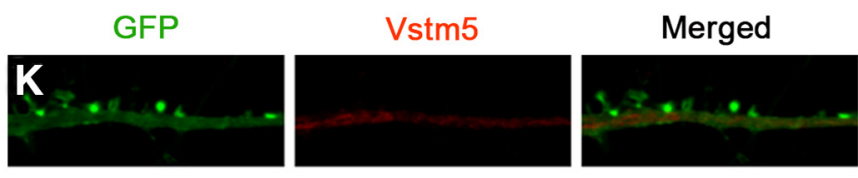

$\beta$-actin::GFP + Vstm5/pcDNA3.1
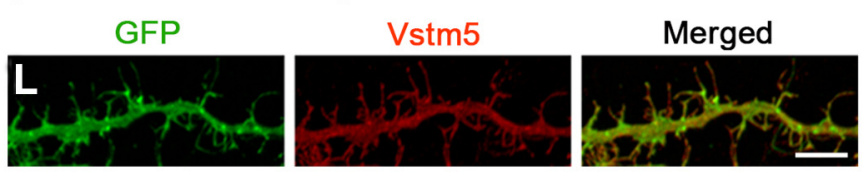
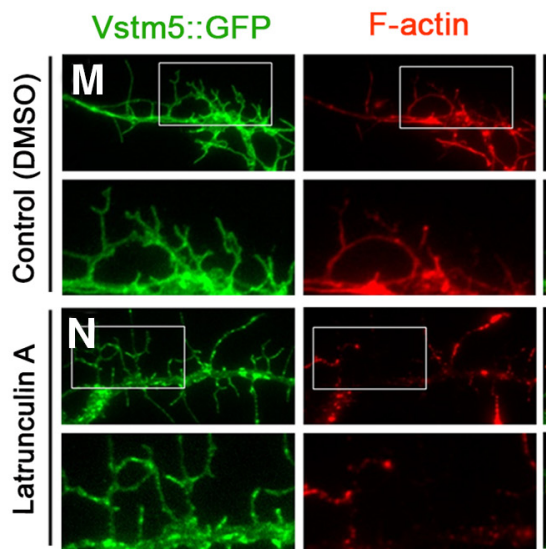

Merged

Figure 7. Vstm5 regulates actin distribution and synapse remodeling in mouse hippocampal neurons. $\boldsymbol{A}-\boldsymbol{D}, \boldsymbol{F}-\boldsymbol{I}$, Transfected neurons were immunostained using PSD-95 (blue) and synaptophysin-1 (red) antibodies and palloidin $(\boldsymbol{F}-\boldsymbol{I})$ and the resulting images were merged. $\boldsymbol{E}$, Quantification of PSD-95 and SYP-1 colocalized puncta on dendritic spines (control, $n=15 ; \mathrm{Vstm} 5$, $n=18 ; \operatorname{shScramble,~} n=9$; and shVstm5, $n=15$ ). $\boldsymbol{J}$, F-actin fluorescence intensity ratios (dendritic spine/dendritic shaft) in $\boldsymbol{F}-\boldsymbol{I}$ (control, $n=11 ; \mathrm{Vstm} 5, n=11 ; \mathrm{shS}$ cramble, $n=18$; and shVstm5, $n=15) . K, L$, Neurons at DIV10 were transfected with $\beta$-actin::GFP plus pcDNA3.1 or $\beta$-actin::GFP plus Vstm5/pcDNA3.1, and immunostained with anti-GFP (green) and anti-Vstm5 (red) antibodies at DIV21. $\boldsymbol{M}, \mathbf{N}, \mathrm{Vstm5}:$ :GFP-expressing neurons at 14 DIV were treated with DMSO $(\boldsymbol{M})$ or $5 \mu \mathrm{M}$ latrunculin A $(\boldsymbol{N})$ for $24 \mathrm{~h}$ and stained with an anti-GFP (green) antibody and phalloidin (red). A high-magnification view of the region enclosed by the rectangle is shown below each image. Error bars indicate SEM. ${ }^{*} p<0.05,{ }^{* *} p<0.01,{ }^{* * *} p<0.001$. Scale bars: (in $\left.\boldsymbol{D}, \boldsymbol{I}\right) \boldsymbol{A}-\boldsymbol{D}, \boldsymbol{F}-\boldsymbol{I}$ and (in L) $K, L, 5 \mu \mathrm{m}$; (in $N) M, N$, low, $10 \mu \mathrm{m}$; high, $5 \mu \mathrm{m}$.

alters the subneuronal distribution of F-actin and the morphology of dendritic protrusions.

Vstm5 is crucial for neuronal migration in vivo

To determine the function of Vstm5 in vivo, we introduced WT Vstm5 and shVstm5 into developing mouse embryos. In utero electroporation was performed on E14.5, a period when neurons destined for the upper cortical plate (UCP) are born. The brains of newborn mice were fixed at P1 for immunostaining with a GFP antibody and DAPI. For quantification, we subdivided the cerebral cortex into 10 layers including the MZ (marginal zone), dCP (dense cortical plate), layers 5/6, IZ (intermediate zone), and SVZ (subventricular zone), based on their DAPI staining patterns (Fig. $8 A-D$ ). Some striking changes were seen in the distribution and morphology of neurons in these different layers. In the control, most of the GFP-expressing neurons arrived at the upper cortical layers, dCP and MZ (Fig. $8 A, E$ ). In contrast, migration of Vstm5-expressing neurons to the 

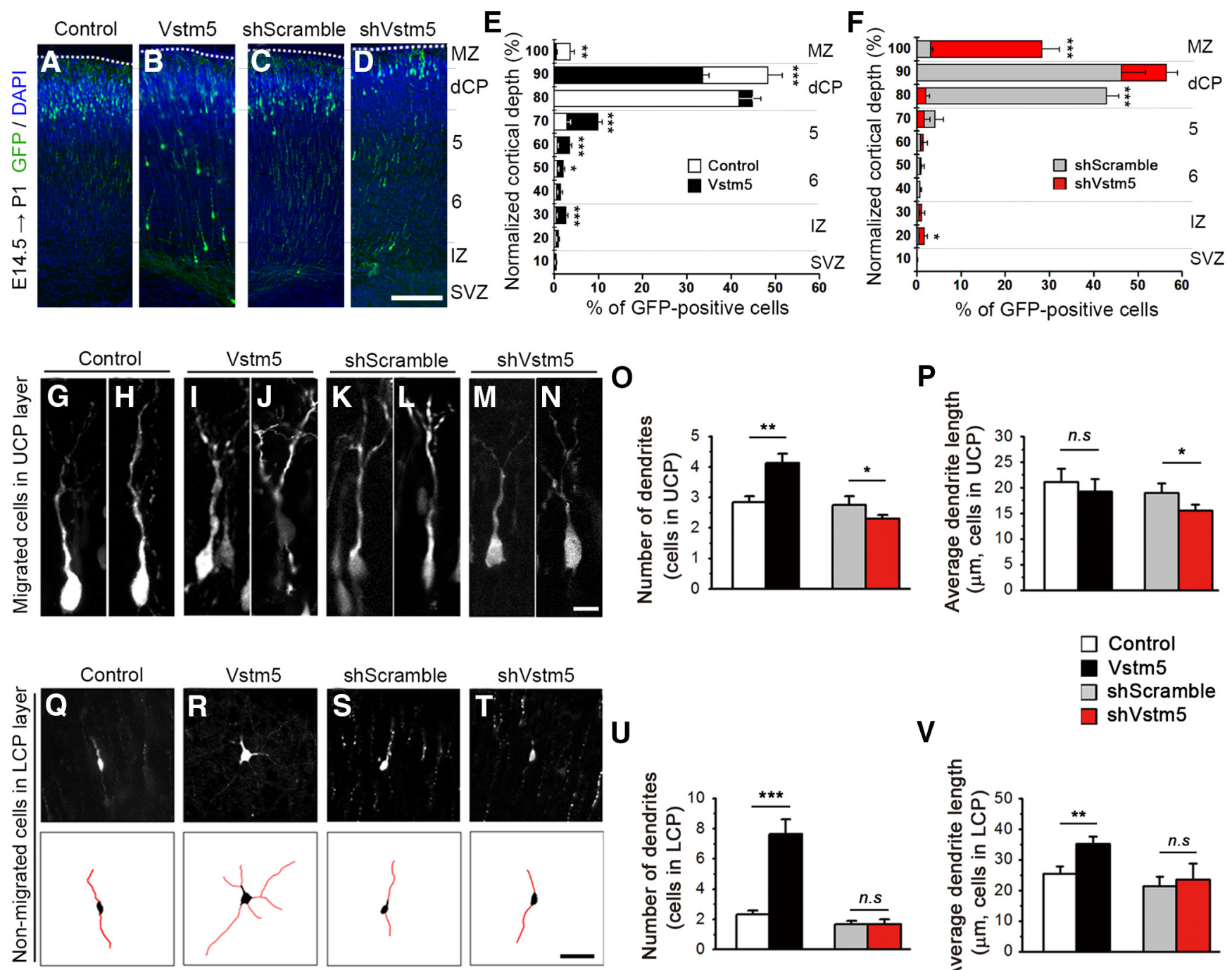

\section{0}
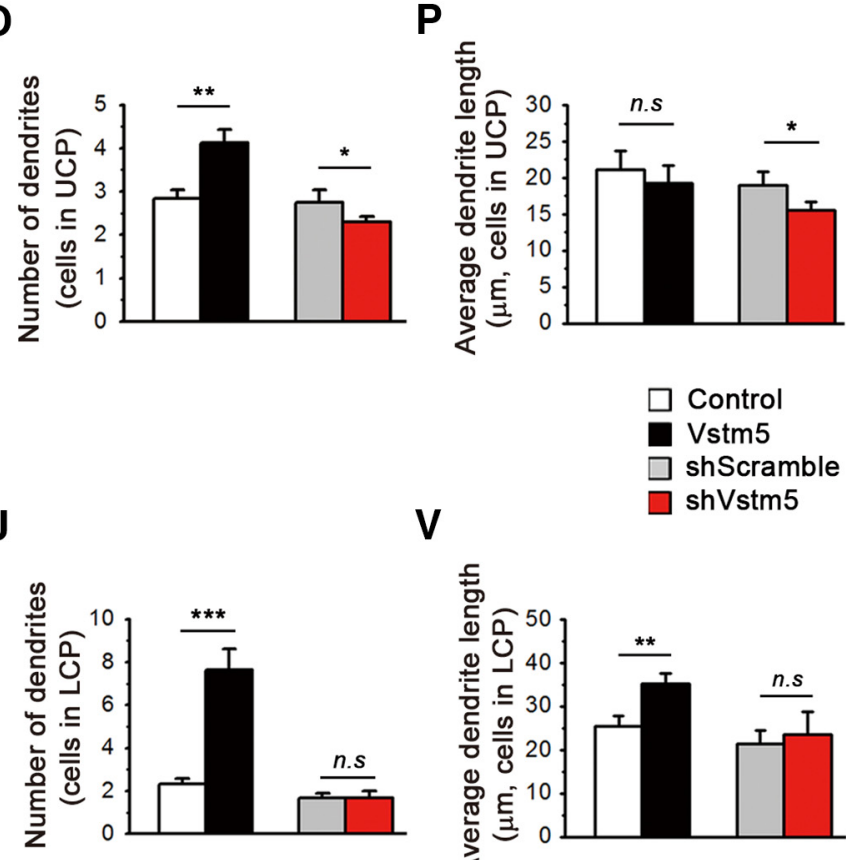
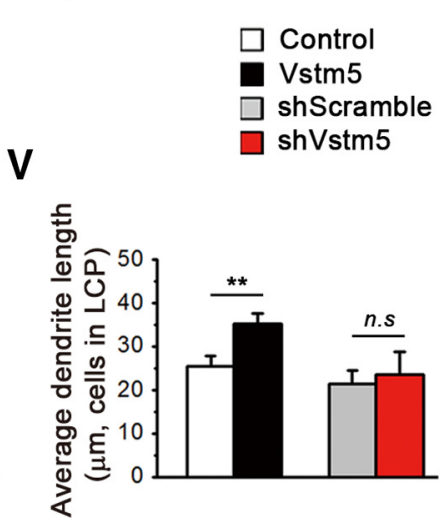

Figure 8. Vstm5 regulates dendrite formation in vivo. A-D, Plasmid DNAs encoding pCAGIG (A), Vstm5/pCAGIG (B), shScramble/pCAG-mir30-IRES-GFP ( $\boldsymbol{C}$, and shVstm5/pCAG-mir30-IRES-GFP (D) were transfected into embryonic mouse cortical neurons at E14.5 using in utero electroporation. Mice were killed on P1. Brain slices were immunostained using an anti-GFP antibody and with DAPI (blue). Dotted lines represent pia. $\boldsymbol{E}, \boldsymbol{F}$, GFP-positive cells in $\boldsymbol{A}-\boldsymbol{D}$ were counted and expressed as the percentage of the total number of GFP-positive cells (GFP, $n=5$ sections; Vstm5, $n=9$ sections; shScramble, $n=9$ sections; and shVstm5, $n=8$ sections). $\mathbf{G}-\boldsymbol{N}$, Confocal images of GFP-positive electroporated cortical neurons in the UCP. $\mathbf{0}, \boldsymbol{P}$, Dendrite number ( $\mathbf{0}$ ) and average length (P) of neurons fully migrated to the UCP (GFP, $n=6 ; V s t m 5, n=7 ;$ shScramble, $n=8 ;$ and shVstm5, $n=11$ ). Q- $\boldsymbol{T}$, Confocal images of nonmigrated cortical neurons in the $L$ CP. Bottom shows traces of unmigrated neurons, with neurites traced in red. $\boldsymbol{U}, \boldsymbol{V}$, Dendrite number ( $\boldsymbol{U}$ ) and average dendrite length ( $\boldsymbol{V}$ in LCP cells (GFP, $n=10 ; \mathrm{Vstm} 5, n=15 ; \operatorname{shScramble,~} n=9$; and shVstm5, $n=5$ ). Error bars indicate SEM. ${ }^{*} p<0.05,{ }^{* *} p<0.01,{ }^{* * *} p<0.001$; ns, no statistical significance. Scale bars: (in $\left.\boldsymbol{D}\right) \boldsymbol{A}-\boldsymbol{D}, 100 \mu \mathrm{m}$; (in $\left.\boldsymbol{N}\right) \mathbf{G}-\boldsymbol{N}, 10 \mu \mathrm{m}$; (in $\boldsymbol{T}$ ) $\mathbf{Q}-\boldsymbol{T}, 40 \mu \mathrm{m}$.

$\mathrm{dCP}$ and $\mathrm{MZ}$ was disrupted, with some cells remaining in the lower cortical layers 5/6 and IZ (Fig. 8B,E). Interestingly, Vstm5-knock-down neurons settled in the MZ closer to the pial side and the corresponding percentage of neurons was decreased in the dense cortical plate, reflecting the overmigration of those neurons (Fig. $8 C, D, F$ ). In the final phase of migration, neurons overexpressing Vstm 5 in the UCP induced excessive branching of the leading processes $(2.83 \pm 0.21 /$ cell for control and $4.13 \pm 0.31 /$ cell for Vstm5; Fig. $8 G-J, O)$. Conversely, Vstm5-knock-down neurons in the UCP resulted in small but significant reductions in both the number of dendrites $(2.75 \pm 0.28 /$ cells for shScramble and $2.31 \pm 0.11 /$ cells for shVstm5) and average dendrite length (Fig. $8 K-P$ ). Next, we traced the nonmigrated neurons in the LCP and analyzed the number of branches in the leading processes. Whereas the number and average length of dendrites were significantly increased in the neurons overexpressing Vstm5 compared with those in control (Fig. 8Q, $R, U, V$ ), no significant changes were found in the neurons expressing shVstm5 compared with shScramble control (Fig. $8 S-V$ ). These results indicate that Vstm 5 controls branching in the leading processes of migrating neurons, and thus affects neuronal migration and differentiation in vivo.

\section{Vstm5 regulates dendritic branching and controls spine morphology in vivo}

We next examined neuronal morphology in the brains of P10 mice that had been subjected to in utero electroporation at E14.5. Whereas shVstm5-expressing neurons overmigrated at a region of the layer 2, Vstm5-expressing nonmigrated cells were found in layer $4 / 5$ of the brain (Fig. $9 A-F$ ), consistent with the results obtained at an earlier stage (P1). We then analyzed the dendritic arborization (within $150 \mu \mathrm{m}$ from the soma) of neurons in layer 2/3, which included basal and apical dendrite regions (Fig. 9G-J). Vstm5-expressing neurons showed a significant increase in dendrite branching in the region between 30 and $45 \mu \mathrm{m}$ (Fig. 

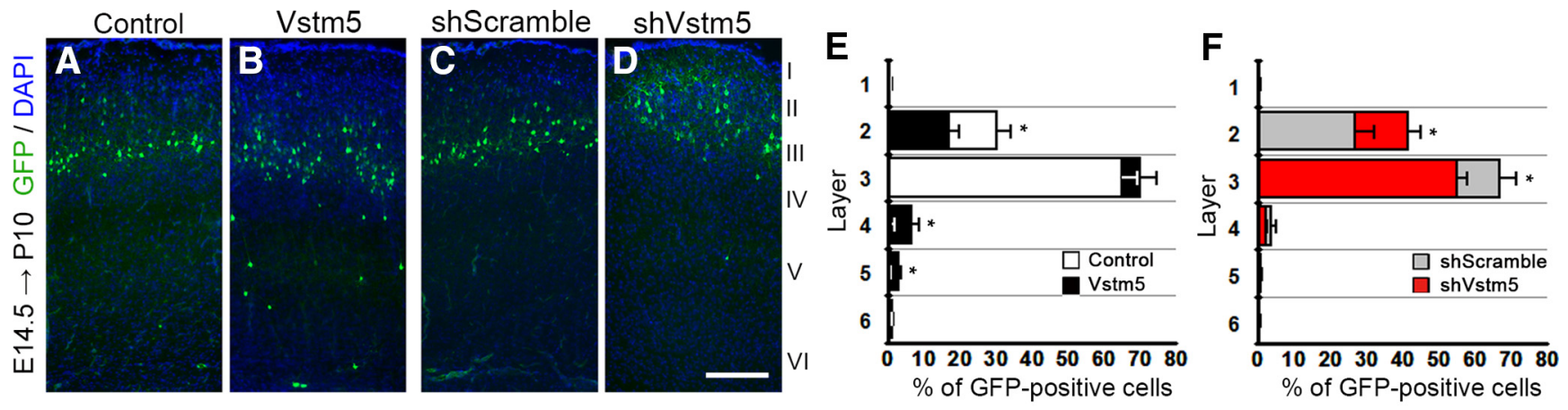
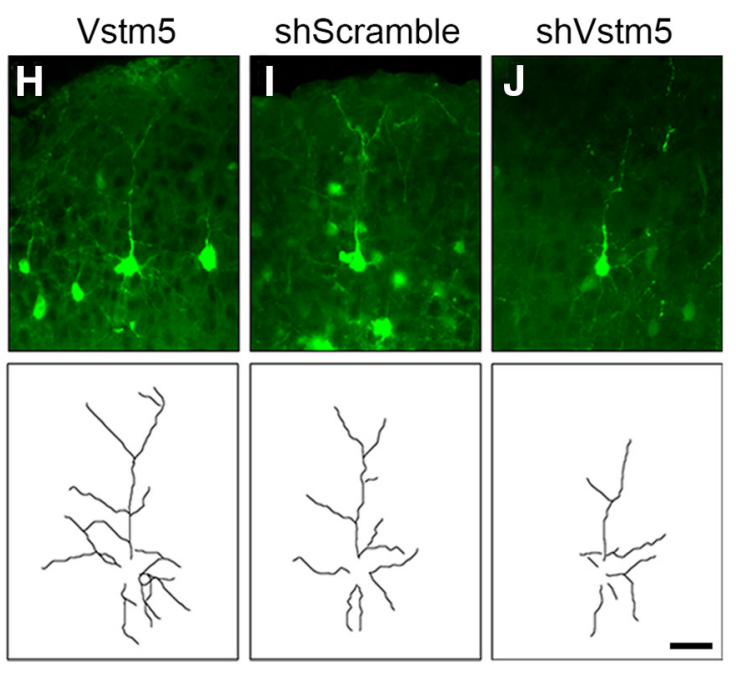
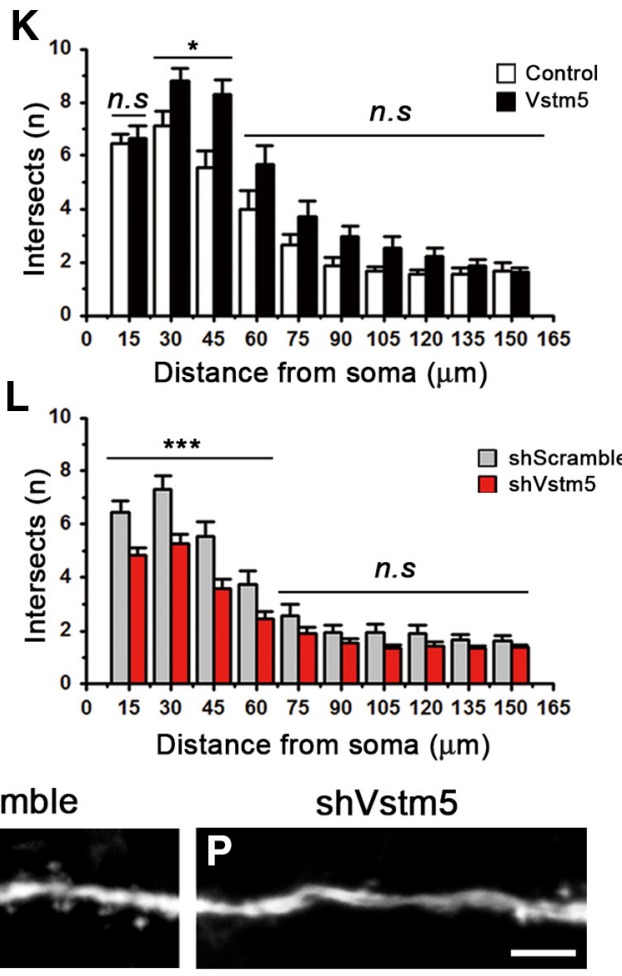

S
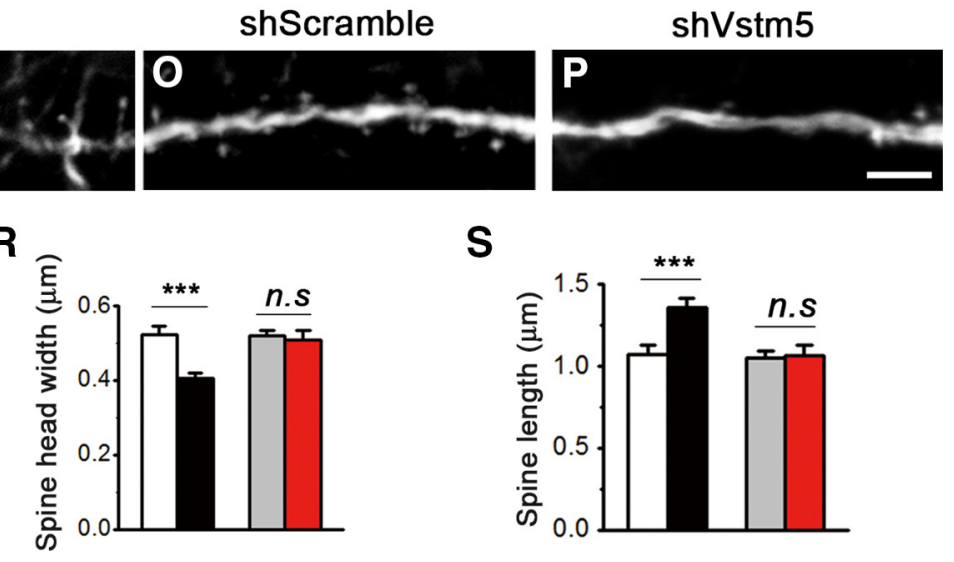

T

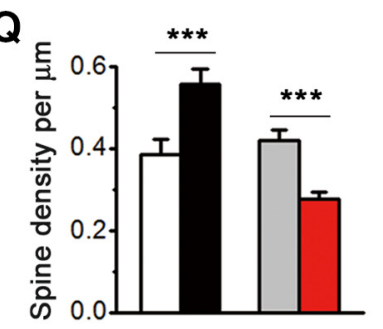

Vstm5

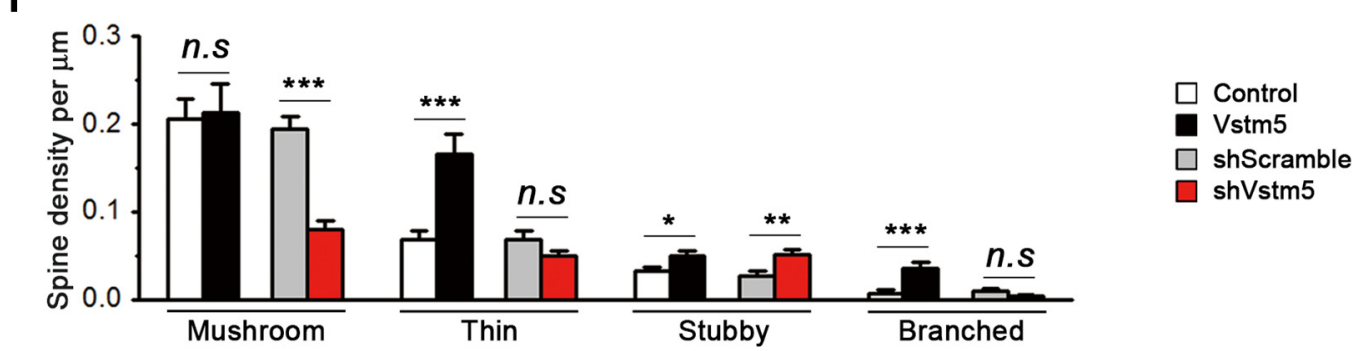

Figure 9. Vstm5 regulates neuronal morphology in vivo. $\boldsymbol{A}-\boldsymbol{D}$, Mouse brains in utero were electroporated at E14 and mice were killed at P10. Coronal sectioned brain slices immunostained with an anti-GFP antibody (green); nuclei are stained with DAPI (blue). $\boldsymbol{E}, \boldsymbol{F}$, Number of GFP-expressing cells in the 6 layers counted as a percentage (\%) of the total number of GFP-positive cells (GFP, $n=$ 4 sections; Vstm5, $n=7$ sections; shScramble, $n=5$ sections; and shVstm5, $n=8$ sections). $\mathbf{G}-\boldsymbol{J}$, Immunostained layer $2 / 3$ neurons are shown with drawing of neurons. $\boldsymbol{K}, \boldsymbol{L}$, Sholl analysis of dendrites from layer $2 / 3$ neurons. Dendritic complexity analyzed from the soma to $150 \mu \mathrm{m}$ in control $(\boldsymbol{G})$ and pyramidal neurons expressing Vstm5 (H), shScramble $(\boldsymbol{I})$, and shVstm5 $(\boldsymbol{J})(\mathrm{GFP}, n=$ 20; Vstm5, $n=25$; shScramble, $n=23$; and shVstm5, $n=21$ ). $\boldsymbol{M}-\boldsymbol{P}$, Immunostained images of basal dendrites in layer $2 / 3$ pyramidal neurons. Q-S, (Figure legend continues.) 
$9 G, H, K)$. In contrast, the expression of shVstm5 markedly reduced the complexity of the dendritic arbor at 15 to $60 \mu \mathrm{m}$ compared with that in the shScramble control (Fig. 9I, J,L). Consistent with the aforementioned results using cultured hippocampal neurons (Fig. $5 N-S$ ), these results demonstrate the importance of Vstm5 in controlling dendritic complexity in vivo as well as in vitro.

Next, we examined the density and morphology of dendritic protrusions in layer $2 / 3$ neurons at high resolution (Fig. $9 M-P$ ). Whereas neurons overexpressing Vstm5 exhibited an increased density of dendritic spines (Fig. 9M,N,Q), Vstm5-knock-down neurons showed much lower levels of spines (Fig. 9O-Q). Moreover, in Vstm5-expressing neurons, the average length of protrusions was increased, with a reduction in spine head width (Fig. 9R,S). For shScramble- and shVstm5-expressing neurons, there were no significant differences in head width or spine length (Fig. $9 R, S$ ). In terms of spine morphology, the densities of thin, stubby, and branched spines were markedly increased in Vstm5-induced dendritic spines without any significant change in the number of mushroom-type spines compared with those in controls (Fig. 9T). In Vstm5-knockdown neurons, however, the density of mushroom spines was markedly decreased compared with that in control neurons (Fig. 9T). In general, the results of the density and morphology of spines in brains of P10 mice were well correlated with those from cultured hippocampal neurons in vitro. Together, these results further indicate that Vstm5 induces dendritic protrusions and promotes the in vivo formation of unstable neuronal spines such as the thin, stubby, and branched types.

\section{Discussion}

CAMs are transmembrane glycoproteins expressed on the plasma membrane, performing a variety of cellular functions, including immune response, inflammation, and neuronal development (Bacus et al., 1994; Bruijn and de Heer, 1995; Haapasalmi et al., 1995). Four groups of CAMs have been identified: the integrin family, the Ig superfamily (IgSF), selectins, and cadherins (Shapiro et al., 2007; Takeichi, 2007). While searching for novel CNS CAMs, we identified a small transmembrane protein with an unannotated function called Vstm5. A series of preliminary transfection assays revealed that Vstm5 strongly induced membrane protrusions resembling filopodia in various cell lines. Intrigued by its small size $(19.5 \mathrm{kDa})$ and spatiotemporal expression pattern in the brain, we investigated the biochemical characteristics and functional roles of Vstm 5 in the CNS.

Belonging to the IgSF, Vstm5 has the simplest structural arrangement among any known IgSF CAMs. Vstm5 shows a notable resemblance to myelin protein zero (P0) in membrane topology and domain arrangement. As a major structural protein almost exclusively expressed in the peripheral nervous system, P0 is involved in the formation and maintenance of the myelin sheath (Shapiro et al., 1996). Unlike P0, however, Vstm5 is highly expressed in the CNS and a few other tissues, such as the stomach, kidney, and intestines. Although Vstm5 is highly conserved in different species, especially in mammals (Fig. $1 B, D$ ), it is distinct from other members of the VSTM family in terms of overall amino acid sequence and domain structure (data not shown).

$\leftarrow$

(Figure legend continued.) Spine density $(\mathbf{Q})$, head width $(\boldsymbol{R})$, and length $(\boldsymbol{S})$ in transfected neurons. $\boldsymbol{T}$, Spine density in dendritic spines classified based on shape (GFP, $n=9 ; \mathrm{Vstm} 5, n=$ 11 ; shScramble, $n=13$; and shVstm5, $n=16$ ). Error bars indicate SEM. ${ }^{*} p<0.05,{ }^{* *} p<$ $0.01,{ }^{* * *} p<0.001$; ns, no statistical significance. Scale bars: (in $\left.\boldsymbol{D}\right) \boldsymbol{A}-\boldsymbol{D}, 100 \mu \mathrm{m}$; (in $\left.\boldsymbol{J}\right) \boldsymbol{G}-\boldsymbol{J}, 50$ $\mu \mathrm{m} ;$ (in $\boldsymbol{P}) \boldsymbol{M}-\boldsymbol{P}, 5 \mu \mathrm{m}$.
Recently, two other VSTM family proteins, VSTM1 and VSTM3 (known as TIGIT and WUCAM, respectively), were shown to be involved in the immune response (Steevels et al., 2010; Levin et al., 2011; Guo et al., 2012).

Considering the molecular size and simple domain structure of Vstm5, it is surprising and intriguing to observe the dramatic effects of Vstm5 expression in various mammalian cell lines. Vstm5 induces, not only highly mobile filopodia-like structures, but also the dynamic movement of membrane ruffles and rapid trafficking of vesicle-like structures, for example, in COS-7 cells transfected with GFP-tagged Vstm5 (Fig. 3N, Movie 2). These Vstm5-induced changes in membrane dynamics raised a fundamental question: how could this small protein induce such striking changes in membrane structure and dynamics in various cell lines of different origins? Many IgCAMs are now known to interact with cellular proteins via their short cytoplasmic $\mathrm{C}$ termini (Juliano, 2002). The juxtamembrane region of Vstm5, containing multiple basic residues, is critical for the induction of membrane filopodia (Fig. 4A, F, G, K, L). Because Rho GTPases are key regulators of cytoskeletal dynamics and affect membrane dynamics (Heasman and Ridley, 2008), we examined the role of Rho GTPases in Vstm5-induced membrane protrusion formation. We generated shRNAs against Cdc42 and Rac1 and performed knock-down experiments (Fig. $4 N-U$ ) and Cdc42/Rac1 activation assay (Fig. $4 V-X$ ). We found that Vstm5-induced dendritic filopodia formation involves the $\mathrm{Cdc} 42$ pathway and the juxatransmembrane region of Vstm5 may activate Cdc42 to induce membrane filopodia. Vstm5 also affected the activity of Rac1. However, Rac1 knock-down did not significantly altered Vstm5induced dendritic filopodia formation (Fig. $4 U, X$ ).

In developing mouse brain, the expression of Vstm 5 increases markedly from E15 to P1 (Fig. 2D), a period corresponding to the early stage of postmitotic neuronal differentiation, when neuronal morphogenesis or synapse formation occurs. Neuronal morphogenesis, such as that involved in dendritic arbor and dendritic spine structure development, is crucial for synaptic integration and plasticity in neural circuits (Spruston, 2008). During dendritic arbor development, the number of dendrites and their branching pattern are strictly correlated with the function of the particular neuron and the geometry of the connections it receives (Urbanska et al., 2012). In developing neurons, we found that suppression of endogenous Vstm5 resulted in lower densities of dendritic filopodia (Fig. $5 K, M$ ) and dendritic complexities (Fig. $5 Q, S$ ), which are required for neuritogenesis (Dent et al., 2007; Heiman and Shaham, 2010). In contrast, overexpression of Vstm5 facilitated dendritic filopodial development (Fig. 4J,L) and dendrite formation and branching (Fig. 5O,R). Therefore, Vstm 5 may regulate dendritic formation in the neuronal differentiation stage and thereby increase the chance of dendrites connecting with the axons of neighboring neurons and creating synaptic connections.

In spinogenesis, dendritic filopodia are abundant and dynamic during the rapid synaptogenesis period (the first postnatal week). However, their number and motility are gradually reduced, and thin and mushroom spines begin to emerge during the second postnatal week (Fiala et al., 1998). We observed that the time course of spinogenesis was inversely correlated with the expression level of Vstm5. Spine density is significantly increased by the expression of Vstm5, mainly due to more unstable, immature forms of spines such as thin, stubby, and branched types (Fig. $6 J, N)$. These phenotypes are shown in both early developing brains and synaptic-transmission-blocked mature brains (Petrak et al., 2005). This suggests that the level of Vstm5 must be tightly 
controlled because Vstm 5 can interfere with spinogenesis by acting as a negative regulator, preventing spine formation. Interestingly, the total spine density and number of mushroom spines are decreased in Vstm5-knock-down neurons (Fig. 6P, Q,T), suggesting that dendritic filopodia are essential intermediate structures during spinogenesis and synapse formation. Therefore, it is conceivable that Vstm5 is involved in the structural plasticity of neurons during CNS development.

Spine structure and its associated synapse regulate the morphological synaptic plasticity at excitatory synapses, which is related to short- and long-term memory (Calverley and Jones, 1990; Muller et al., 2000). In early synaptogenesis, dendritic filopodia are known to actively initiate contacts with nearby axonal shafts and presynaptic and postsynaptic molecules are redistributed and thereafter evolve into dendritic spines (Ziv and Smith, 1996). Synaptic plasticity is strongly correlated with the distribution and organization of subcellular molecules of spines such as IP3 receptors, Homer, GKAP, F-actin, PSD-95, NR1, and GluR2/3 (Sala et al., 2001). In the present study, we found that the density of excitatory synapses was increased by Vstm 5 expression (Fig. $7 A, B, E$ ), but decreased in Vstm5-knock-down neurons (Fig. $7 C-E)$, suggesting that Vstm5 may be an important regulator of differentiation in excitatory synapses. In addition, dendritic spine plasticity is associated with actin cytoskeleton distribution and induces a redistribution of presynaptic sites (Matus et al., 1982; Matus et al., 2000; Pilo Boyl and Witke, 2014). We observed that Vstm5 induced reorganization of F-actin and increased formation of unstable spines such as thin or branched types. Unstable spine structures are critical for initial synaptic contact with presynaptic sites and Vstm 5 may also drive synaptic plasticity by regulating F-actin composition and altering spine morphology (Fig. $7 F-J$ ). Furthermore, a recent study reported that Vstm5 is expressed in ventral spinal cord (motor neurons) and sensory neurons (Gu et al., 2015). Therefore, Vstm 5 may also be involved in establishing the synaptic specificity of sensory-motor circuits.

During the sequential steps of migration, neurons constantly change their morphology, starting with multipolar migration in the IZ, progressing to bipolar radial migration in the cortical plate, and ending at the terminal translocation stage in which neurons develop more branches at the outermost region of the cortical plate (Nadarajah et al., 2001; Nadarajah and Parnavelas, 2002; Ohshima et al., 2007). Vstm5 appears to become involved starting with radial migration because changes in Vstm5 levels altered the distribution of neurons in the cortical plate (Figs. $8 A, B, E, 9 A, B, E)$. The level of Vstm5 also correlated well with the number of branches in the leading processes of migrating neurons, showing more branches in Vstm5-overexpressing and less in Vstm5-knock-down neurons (Fig. 8G-V). Furthermore, Vstm5 altered dendritic complexity to affect neuronal migration during corticogenesis (Fig. 9A-L). During the transition from radial migration to terminal translocation, multiple branches appear in the leading process, which contributes to the early development of apical dendrite morphogenesis (Chai et al., 2015). Therefore, it is plausible that the expression level of Vstm 5 affects neurite formation at the leading processes of migrating neurons to facilitate dendrite formation and integration of neurons into the appropriate laminae. This conjecture is in good agreement with the potential roles of balanced Vstm 5 expression in dendrite and synapse formation during brain development.

In a recent study, Vstm5 was identified as one of the target genes responsible for the differential response to the treatment of major depressive disorder (MDD; Biernacka et al., 2015). The genetic variations of Vstm5 among patients with MDD were re- lated to the variable effects of selective serotonin reuptake inhibitors in MDD treatment (Anderson et al., 2012). According to studies on MDD and a rodent stress model, the size of the depression-related brain regions decreases and neuronal atrophy appears (reduction in the length and branching of apical dendrites) with reduced synapses (Rajkowska et al., 1999; Stockmeier et al., 2004; Duman and Aghajanian, 2012). Therefore, the biochemical and physiological characteristics of Vstm 5 presented in the present study may provide insight for the altered brain morphology observed in stress-induced depression and MDD.

In summary, we have identified a small but novel membrane protein, Vstm5, which markedly influenced the structure and dynamics of the cell membrane. Vstm5 increased dendritic complexity and synapse formation in vivo as well as in vitro and controlled neuronal migration in the brain. Therefore, Vstm5 may play crucial roles in formation of dendritic structure and synaptic connectivity in the vertebrate nervous system.

\section{References}

Alfonso J, Fernández ME, Cooper B, Flugge G, Frasch AC (2005) The stressregulated protein $\mathrm{M} 6 \mathrm{a}$ is a key modulator for neurite outgrowth and filopodium/spine formation. Proc Natl Acad Sci U S A 102:17196-17201. CrossRef Medline

Anderson HD, Pace WD, Libby AM, West DR, Valuck RJ (2012) Rates of 5 common antidepressant side effects among new adult and adolescent cases of depression: a retrospective US claims study. Clin Ther 34: 113-123. CrossRef Medline

Bacus SS, Zelnick CR, Chin DM, Yarden Y, Kaminsky DB, Bennington J, Wen D, Marcus JN, Page DL (1994) Medullary carcinoma is associated with expression of intercellular adhesion molecule-1. Implication to its morphology and its clinical behavior. Am J Pathol 145:1337-1348. Medline

Bhatt DH, Zhang S, Gan WB (2009) Dendritic spine dynamics. Annu Rev Physiol 71:261-282. CrossRef Medline

Biernacka JM et al. (2015) The International SSRI Pharmacogenomics Consortium (ISPC): a genome-wide association study of antidepressant treatment response. Transl Psychiatry 5:e553. CrossRef Medline

Bruijn JA, de Heer E (1995) Adhesion molecules in renal diseases. Lab Invest 72:387-394. Medline

Burgess A, Wainwright SR, Shihabuddin LS, Rutishauser U, Seki T, Aubert I (2008) Polysialic acid regulates the clustering, migration, and neuronal differentiation of progenitor cells in the adult hippocampus. Dev Neurobiol 68:1580-1590. CrossRef Medline

Calverley RK, Jones DG (1990) Contributions of dendritic spines and perforated synapses to synaptic plasticity. Brain Res Brain Res Rev 15:215-249. CrossRef Medline

Carlson B, Soderling SH (2009) Mechanisms of cellular protrusions branch out. Dev Cell 17:307-309. CrossRef Medline

Chai X, Fan L, Shao H, Lu X, Zhang W, Li J, Wang J, Chen S, Frotscher M, Zhao S (2015) Reelin induces branching of neurons and radial glial cells during corticogenesis. Cereb Cortex 25:3640-3653. CrossRef Medline

Chang S, De Camilli P (2001) Glutamate regulates actin-based motility in axonal filopodia. Nat Neurosci 4:787-793. CrossRef Medline

Chothia C, Jones EY (1997) The molecular structure of cell adhesion molecules. Annu Rev Biochem 66:823-862. CrossRef Medline

Crespi A, Ferrari I, Lonati P, Disanza A, Fornasari D, Scita G, Padovano V, Pietrini G (2012) LIN7 regulates the filopodium- and neurite-promoting activity of IRSp53. J Cell Sci 125:4543-4554. CrossRef Medline

da Silva JS, Dotti CG (2002) Breaking the neuronal sphere: regulation of the actin cytoskeleton in neuritogenesis. Nat Rev Neurosci 3:694-704. CrossRef Medline

Dent EW, Kwiatkowski AV, Mebane LM, Philippar U, Barzik M, Rubinson DA, Gupton S, Van Veen JE, Furman C, Zhang J, Alberts AS, Mori S, Gertler FB (2007) Filopodia are required for cortical neurite initiation. Nat Cell Biol 9:1347-1359. CrossRef Medline

Duman RS, Aghajanian GK (2012) Synaptic dysfunction in depression: potential therapeutic targets. Science 338:68-72. CrossRef Medline

Fiala JC, Feinberg M, Popov V, Harris KM (1998) Synaptogenesis via dendritic filopodia in developing hippocampal area CA1. J Neurosci 18: 8900-8911. Medline

Fiala JC, Spacek J, Harris KM (2002) Dendritic spine pathology: cause or 
consequence of neurological disorders? Brain Res Brain Res Rev 39:2954. CrossRef Medline

Gu Z, Imai F, Kim IJ, Fujita H, Katayama Ki, Mori K, Yoshihara Y, Yoshida Y (2015) Expression of the immunoglobulin superfamily cell adhesion molecules in the developing spinal cord and dorsal root ganglion. PLoS One 10:e0121550. CrossRef Medline

Guerrier S, Coutinho-Budd J, Sassa T, Gresset A, Jordan NV, Chen K, Jin WL, Frost A, Polleux F (2009) The F-BAR domain of srGAP2 induces membrane protrusions required for neuronal migration and morphogenesis. Cell 138:990-1004. CrossRef Medline

Guo X, Zhang Y, Wang P, Li T, Fu W, Mo X, Shi T, Zhang Z, Chen Y, Ma D, Han W (2012) VSTM1-v2, a novel soluble glycoprotein, promotes the differentiation and activation of Th17 cells. Cell Immunol 278:136-142. CrossRef Medline

Haapasalmi K, Mäkelä M, Oksala O, Heino J, Yamada KM, Uitto VJ, Larjava H (1995) Expression of epithelial adhesion proteins and integrins in chronic inflammation. Am J Pathol 147:193-206. Medline

Harris KM, Jensen FE, Tsao B (1992) Three-dimensional structure of dendritic spines and synapses in rat hippocampus (CA1) at postnatal day 15 and adult ages: implications for the maturation of synaptic physiology and long-term potentiation. J Neurosci 12:2685-2705. Medline

Heasman SJ, Ridley AJ (2008) Mammalian Rho GTPases: new insights into their functions from in vivo studies. Nat Rev Mol Cell Biol 9:690-701. CrossRef Medline

Heiman MG, Shaham S (2010) Twigs into branches: how a filopodium becomes a dendrite. Curr Opin Neurobiol 20:86-91. CrossRef Medline

Hodges RS, Heaton RJ, Parker JM, Molday L, Molday RS (1988) Antigenantibody interaction. Synthetic peptides define linear antigenic determinants recognized by monoclonal antibodies directed to the cytoplasmic carboxyl terminus of rhodopsin. J Biol Chem 263:11768-11775. Medline

Hotulainen P, Hoogenraad CC (2010) Actin in dendritic spines: connecting dynamics to function. J Cell Biol 189:619-629. CrossRef Medline

Juliano RL (2002) Signal transduction by cell adhesion receptors and the cytoskeleton: functions of integrins, cadherins, selectins, and immunoglobulin-superfamily members. Annu Rev Pharmacol Toxicol 42:283323. CrossRef Medline

Kadowaki M, Nakamura S, Machon O, Krauss S, Radice GL, Takeichi M (2007) N-cadherin mediates cortical organization in the mouse brain. Dev Biol 304:22-33. CrossRef Medline

Kayser MS, Nolt MJ, Dalva MB (2008) EphB receptors couple dendritic filopodia motility to synapse formation. Neuron 59:56-69. CrossRef Medline

Levin SD et al. (2011) Vstm3 is a member of the CD28 family and an important modulator of T-cell function. Eur J Immunol 41:902-915. CrossRef Medline

Lin YL, Lei YT, Hong CJ, Hsueh YP (2007) Syndecan-2 induces filopodia and dendritic spine formation via the neurofibromin-PKA-Ena/VASP pathway. J Cell Biol 177:829-841. CrossRef Medline

Luo L (2002) Actin cytoskeleton regulation in neuronal morphogenesis and structural plasticity. Annu Rev Cell Dev Biol 18:601-635. CrossRef Medline

Matsuno H, Okabe S, Mishina M, Yanagida T, Mori K, Yoshihara Y (2006) Telencephalin slows spine maturation. J Neurosci 26:17761786. CrossRef Medline

Mattila PK, Lappalainen P (2008) Filopodia: molecular architecture and cellular functions. Nat Rev Mol Cell Biol 9:446-454. CrossRef Medline

Matus A, Ackermann M, Pehling G, Byers HR, Fujiwara K (1982) High actin concentrations in brain dendritic spines and postsynaptic densities. Proc Natl Acad Sci U S A 79:7590-7594. CrossRef Medline

Matus A, Brinkhaus H, Wagner U (2000) Actin dynamics in dendritic spines: a form of regulated plasticity at excitatory synapses. Hippocampus 10:555-560. Medline

Muller D, Toni N, Buchs PA (2000) Spine changes associated with longterm potentiation. Hippocampus 10:596-604. Medline

Nadarajah B, Parnavelas JG (2002) Modes of neuronal migration in the developing cerebral cortex. Nat Rev Neurosci 3:423-432. CrossRef Medline

Nadarajah B, Brunstrom JE, Grutzendler J, Wong RO, Pearlman AL (2001) Two modes of radial migration in early development of the cerebral cortex. Nat Neurosci 4:143-150. CrossRef Medline

Niessen CM, Leckband D, Yap AS (2011) Tissue organization by cadherin adhesion molecules: dynamic molecular and cellular mechanisms of morphogenetic regulation. Physiol Rev 91:691-731. CrossRef Medline
Ohshima T, Hirasawa M, Tabata H, Mutoh T, Adachi T, Suzuki H, Saruta K, Iwasato T, Itohara S, Hashimoto M, Nakajima K, Ogawa M, Kulkarni AB, Mikoshiba K (2007) Cdk5 is required for multipolar-to-bipolar transition during radial neuronal migration and proper dendrite development of pyramidal neurons in the cerebral cortex. Development 134:22732282. CrossRef Medline

Paddison PJ, Cleary M, Silva JM, Chang K, Sheth N, Sachidanandam R, Hannon GJ (2004) Cloning of short hairpin RNAs for gene knock-down in mammalian cells. Nat Methods 1:163-167. CrossRef Medline

Pak DT, Yang S, Rudolph-Correia S, Kim E, Sheng M (2001) Regulation of dendritic spine morphology by SPAR, a PSD-95-associated RapGAP. Neuron 31:289-303. CrossRef Medline

Petrak LJ, Harris KM, Kirov SA (2005) Synaptogenesis on mature hippocampal dendrites occurs via filopodia and immature spines during blocked synaptic transmission. J Comp Neurol 484:183-190. CrossRef Medline

Pilo Boyl P, Witke W (2014) Small, smaller ... dendritic spine. EMBO J

Portera-Cailliau C, Pan DT, Yuste R (2003) Activity-regulated dynamic behavior of early dendritic protrusions- evidence for different types of dendritic filopodia. J Neurosci 23:7129-7142. Medline

Rajkowska G, Miguel-Hidalgo JJ, Wei J, Dilley G, Pittman SD, Meltzer HY, Overholser JC, Roth BL, Stockmeier CA (1999) Morphometric evidence for neuronal and glial prefrontal cell pathology in major depression. Biol Psychiatry 45:1085-1098. CrossRef Medline

Ryan XP, Alldritt J, Svenningsson P, Allen PB, Wu GY, Nairn AC, Greengard P (2005) The Rho-specific GEF Lfc interacts with neurabin and spinophilin to regulate dendritic spine morphology. Neuron 47:85-100. CrossRef Medline

Sala C, Piëch V, Wilson NR, Passafaro M, Liu G, Sheng M (2001) Regulation of dendritic spine morphology and synaptic function by Shank and Homer. Neuron 31:115-130. CrossRef Medline

Shapiro L, Doyle JP, Hensley P, Colman DR, Hendrickson WA (1996) Crystal structure of the extracellular domain from $\mathrm{P} 0$, the major structural protein of peripheral nerve myelin. Neuron 17:435-449. CrossRef Medline

Shapiro L, Love J, Colman DR (2007) Adhesion molecules in the nervous system: structural insights into function and diversity. Annu Rev Neurosci 30:451-474. CrossRef Medline

Spruston N (2008) Pyramidal neurons: dendritic structure and synaptic integration. Nat Rev Neurosci 9:206-221. CrossRef Medline

Steevels TA, Lebbink RJ, Westerlaken GH, Coffer PJ, Meyaard L (2010) Signal inhibitory receptor on leukocytes-1 is a novel functional inhibitory immune receptor expressed on human phagocytes. J Immunol 184:47414748. CrossRef Medline

Stockmeier CA, Mahajan GJ, Konick LC, Overholser JC, Jurjus GJ, Meltzer HY, Uylings HB, Friedman L, Rajkowska G (2004) Cellular changes in the postmortem hippocampus in major depression. Biol Psychiatry 56: 640-650. CrossRef Medline

Tabata H, Nakajima K (2001) Efficient in utero gene transfer system to the developing mouse brain using electroporation: visualization of neuronal migration in the developing cortex. Neuroscience 103:865-872. CrossRef Medline

Takeichi M (2007) The cadherin superfamily in neuronal connections and interactions. Nat Rev Neurosci 8:11-20. CrossRef Medline

Urbanska M, Swiech L, Jaworski J (2012) Developmental plasticity of the dendritic compartment: focus on the cytoskeleton. Adv Exp Med Biol 970:265-284. CrossRef Medline

Wood W, Martin P (2002) Structures in focus-filopodia. Int J Biochem Cell Biol 34:726-730. CrossRef Medline

Woolf PJ, Linderman JJ (2003) Self organization of membrane proteins via dimerization. Biophys Chem 104:217-227. CrossRef Medline

Xie Z, Photowala H, Cahill ME, Srivastava DP, Woolfrey KM, Shum CY, Huganir RL, Penzes P (2008) Coordination of synaptic adhesion with dendritic spine remodeling by AF-6 and kalirin-7. J Neurosci 28:60796091. CrossRef Medline

Yoshihara Y, De Roo M, Muller D (2009) Dendritic spine formation and stabilization. Curr Opin Neurobiol 19:146-153. CrossRef Medline

Ziv NE, Smith SJ (1996) Evidence for a role of dendritic filopodia in synaptogenesis and spine formation. Neuron 17:91-102. CrossRef Medline

Zuo Y, Lin A, Chang P, Gan WB (2005) Development of long-term dendritic spine stability in diverse regions of cerebral cortex. Neuron 46: 181-189. CrossRef Medline 